\title{
La diète parfaite d'un lettré retiré sous les Song du Sud
}

\author{
Françoise Sabban'
}

Dans la Chine antique, société où la consommation de viande, toujours valorisée, connotait l'aisance et un certain statut social ${ }^{2}$, consentir à manger maigre hors de toute nécessité économique avait une signification forte. Certains événements de la vie sociale, religieuse ou privée imposaient en principe un changement de régime alimentaire, bianshi selon les termes mêmes de Confucius ${ }^{3}$, qui devait se concrétiser notamment par le jeûne et le renoncement aux nourritures carnées. Ce «changement » de diète

1 Françoise Sabban est Directrice d'études à l'EHESS. Que Catherine Despeux et Frédéric Obringer trouvent ici l'expression de ma reconnaissance pour leur aide généreuse dans l'identification de certaines maladies et affections mentionnées dans le texte. Je remercie également Yves Chevrier et Sylvie Pasquet pour leur lecture attentive et la pertinence de leurs remarques. Une première version de cet article a été présentée lors d'un colloque tenu au Needham Research Institute (Cambridge), en mars 1995.

2 Cf. F. Sabban, "La viande en Chine : imaginaire et usages culinaires ", Anthropozoologica, 18, 1993, p. 79-90 ( Les animaux dans la culture chinoise ").

3 Cf. Lunyu, « Xiangdang ». Anne Cheng traduit : « il change de régime », dans les Entretiens de Confucius, Paris, Le Seuil, 1981, p. 83/7. 


\section{Françoise Sabban}

répondait à une injonction formelle à laquelle tout homme de bien devait se conformer, sous peine d'irrévérence, et sa périodicité avait de plus un rôle de régulation sociale, puisqu'elle symbolisait de manière concrète les rythmes calendaires et le passage du temps à l'échelle de l'existence humaine.

Tout autre est l'interprétation que l'on peut donner des privations de nourriture, de l'abstinence de certains aliments ou de l'adoption d'une diète maigre ou végétarienne lorsque de telles pratiques furent ordonnées dans le cadre du bouddhisme et du taoïsme religieux. Ces comportements anomiques relevaient de convictions actualisées sous forme de choix individuels, dont les justifications sont d'ordre moral ou hygiéniste, alors que le changement alimentaire prôné par Confucius revêt une fonction essentiellement rituelle.

Bien qu'il soit difficile de tracer une filiation directe entre le changement de diète à la Confucius et ces derniers phénomènes qui prirent de l'ampleur avec l'essor du taoïsme religieux et du bouddhisme au cours des dix premiers siècles de notre ère, on peut émettre l'hypothèse que l'instauration de nouvelles règles d'abstinence alimentaire fut facilitée par une habitude fort ancienne de jeûnes périodiques ${ }^{4}$. Dans un premier temps les restrictions ne furent très certainement appliquées avec rigueur que par des religieux ou de fervents croyants nécessairement peu nombreux, puis elles se sont progressivement infiltrées dans les us et coutumes ordinaires, acquérant ainsi une forme de légitimité et, plus inattendu, une reconnaissance gastronomique. À preuve, le sens, devenu générique, de l'expression sushi

4 Encore que la situation soit assez confuse, par manque d'informations. Pour les taoïstes, il faut en effet faire la part des comportements individuels et des comportements collectifs, comme le souligne Rolf Stein. Quant aux bouddhistes, "la position canonique n'était pas claire. La défense n'était pas absolue ou n'était pas toujours appliquée. » Ainsi l'empereur Wu des Liang (502-549), fervent bouddhiste lui-même, « a voulu sévir contre le laxisme des moines de l'époque et imposer l'abstinence de viande et d'alcool » en promulgant un édit dans ce sens. Cf. R. Stein, « Conceptions relatives à la nourriture », in « Étude du monde chinois : institutions et concepts », Annuaire du Collège de France, 1973-1974, p. 458. Voir aussi sur le même sujet, Annuaire du Collège de France, 1971-1972 et 1972-1973. 
pour désigner les mets maigres hors de toute imposition religieuse ou rituelle. Le Qimin yaoshu (Techniques essentielles pour le peuple), célèbre traité d'agriculture, compilé au milieu du vi ${ }^{\mathbf{e}}$ siècle, contient une section ainsi intitulée. Or, la partie culinaire de ce texte témoigne sans aucun doute d'une haute gastronomie, tant est raffiné le corpus de ses recettes ${ }^{5}$, celles qui sont labellées sushi n'étant pas les moins recherchées. Il faut d'ailleurs noter que ces recettes sont essentiellement à base de légumes, sans être strictement végétariennes.

Dès lors, il est probable que l'adoption momentanée ou durable d'un régime maigre ou végétarien pour diverses raisons ait été considérée comme une conduite parmi d'autres de la vie en société, sans qu'elle signale pour autant une appartenance religieuse forte ou définie. Les Song semblent avoir joué un rôle charnière dans cette évolution. Les sources disponibles témoignent en effet d'un véritable intérêt pour la nourriture $s u$. Dans le Mengliang lu (Le rêve du bonheur), une chronique décrivant Hangzhou, capitale des Song du Sud, $10 \%$ des mets cités sont qualifiés de $s u^{6}$. Dans ces textes, l'énumération d'aliments, de mets, voire de restaurants qualifiés de $s u$ signifie l'existence reconnue d'une cuisine spécifique qui devait en toute logique avoir ses habitués. Par ailleurs, c'est aussi sous les Song du

5 À propos de ce texte, cf. Francesca Bray, in Joseph Needham (dir.), Science and Civilisation in China, Vol. 6:Biology and Biological Technology, Part 2: Agriculture, Cambridge, Cambridge University Press, 1984, p. 55-59; F. Sabban, "Suivre les temps du ciel" : économie ménagère et gestion du temps dans la Chine ancienne », in Maurice Aymard, Claude Grignon, Françoise Sabban, Le temps de manger. Alimentation, emploi du temps et rythmes sociaux, Paris, Éditions de la Maison des Sciences de l'Homme/Institut National de la Recherche Agronomique, 1993, p. 67-80.

6 Cf. Lin Zhengqiu et al., Zhongguo Songdai caidian gaishu (Présentation générale des mets de la dynastie des Song), Pékin, Zhongguo shipin chubanshe, 1989, p. 19. Voir aussi les autres descriptions des capitales du Nord et du Sud: Dongjing menghua lu (Rêve de la splendeur de la capitale orientale déchue), Ducheng jisheng (Les sites fameux de la capitale), Xihu laoren fansheng lu (Description des sites fameux du Lac de l'Ouest), Wulin jiushi (Choses anciennes de Wulin). Pour cet article j'ai repris, quand cela était possible, les traductions des titres des ouvrages cités dans A Sung Bibliography, cf. note 12. 


\section{Françoise Sabban}

Nord que de grands poètes, contraints à l'exil ou prenant leur retraite à la fin d'une carrière bien remplie, aspirent à vivre une existence sans artifice au nom d'une hygiène, d'une esthétique et d'une morale. Même s'il faut savoir faire la part de ce qui est pure rhétorique chez ces hommes, habiles manieurs de mots, il arrive que certains d'entre eux soient les auteurs de textes justifiant les plaisirs simples de leur vie quotidienne. Cette époque est riche en monographies consacrées à des objets du monde naturel tels le thé, les champignons, les agrumes, etc., ou en brèves professions de foi comme le Benxinzhai shushipu (Recettes de légumes du studio d'un cœur authentique), un petit recueil poétique décrivant vingt mets végétariens, chers à son auteur, et qu'il destine à ses invités ${ }^{7}$, le Shidafu shishi wuguan (Les cinq principes des temps de manger d'un lettré) de Huang Tingjian, un court manifeste pour une vie sobre et réglée 8 . Dans cet ensemble, le Shanjia qinggong (Les vivres simples du montagnard [désormais abrégé $S J Q G]$ ) de Lin Hong' ${ }^{9}$, que nous nous proposons d'étudier à la lumière de ces questions, occupe une place très importante.

7 Voir à ce sujet Shinoda Osamu, Zhongguo shiwu shi yanjiu (Études d'histoire de l'alimentation chinoise), Gao Guilin et al. (trads.), Pékin, Zhongguo shangye chubanshe, 1987, p. 151 ; Wang Xuetai, Zhongguoren de yinshi shijie (Le monde alimentaire des Chinois), Hong Kong, Zhonghua shuju, 1989, p. 223-225, et Huaxia yinshi wenhua (La culture alimentaire de la Chine), Pékin, Zhonghua shuju, 1993, p. 255-259.

8 Sans compter bien évidemment les œuvres poétiques de Huang Tingjian et d'autres poètes comme Su Shi (Su Dongpo) ou Lu You, pour ne citer que les plus grands, qui fourmillent d'indications à ce sujet par le biais d'allusions et de métaphores.

9 Cf. Wu Ke (éd.), Shanjia qinggong, Pékin, Zhongguo shangye chubanshe, 1985 ("Zhongguo pengren guji congkan »). Voir aussi l'édition Ming, par Zhou Lüjing, reprint par Yang Jialuo, Yishu congbian di yi ji, 36, Yinshibulu, 22 zhong, 90 juan, Taipei, Shijie shuju, 1976. Cf. la notice qui est consacrée à cet ouvrage dans Tao Zhengang et Zhang Lianming (éds.), Zhongguo pengren wenxian tiyao (Notices résumées des sources culinaires chinoises), Pékin, Zhongguo shangye chubanshe, 1986, p. 32-33. 


\section{«Les vivres simples du montagnard »}

\section{L'homme}

Nous ne disposons que de peu d'informations sur Lin Hong. Son œuvre est mince, et le $S J Q G$ n'est précédé d'aucune préface permettant de nous éclairer ${ }^{10}$. Né à Quanzhou au Fujian, Lin Hong est connu comme homme de lettres, et aurait vécu dans la région du Lac de l'Ouest à Hangzhou entre 1241 et $1252^{11}$. S'étant donné comme surnom Keshan, Lin revendique sa parenté avec Lin $\mathrm{Bu}$, un poète de Hangzhou ayant vécu de 967 à $1028^{12}$, et passé à la postérité pour s'être retiré sur la montagne Gushan près du Lac de l'Ouest, où, célibataire endurci, il aurait passé le reste de ses jours sans mettre les pieds en ville. Cette conduite exemplaire ainsi que sa poésie aux éclats fulgurants lui valurent le titre posthume de Hejing xiansheng (Sire Hejing), donné par l'empereur Renzong. De fait, dans le $S J Q G$ Lin Hong évoque plusieurs fois son honorable ancêtre en l'appelant Hejing weng ${ }^{13}$ (Seigneur Hejing), et il rappelle sa filiation avec Lin Bu jusqu'à la septième génération dans le Shanjia qingshi (Les choses simples du montagnard) ${ }^{14}$,

10 Les autres textes de Lin Hong répertoriés dans les catalogues bibliographiques sont au nombre de quatre. Il s'agit de (Xin ke) Wenfang tuzan (Éloge et illustration des instruments du lettré), Xinfeng jiufa (Le vin de Xinfeng [en fait inclus dans le $S J Q G]$ ), Rucao jishi (Notes sur les légumes et les simples) et de Shanjia qingshi (Les choses simples du montagnard). Les deux derniers ouvrages sont des textes très courts. Lin Hong est aussi l'auteur de quelques poésies. Cf. Quan Songci (Intégrale des poèmes en vers irréguliers des Song), 5, 3076, et Songshi jishi (Anthologie de poèmes des Song), 73.

11 Cf. Songren zhuanji ziliao suoyin (Index de matériaux biographiques des Song), Taipei, Dingwen shuju yinhang, vol. 2, 1978.

12 Voir sa notice biographique dans Herbert Franke (éd.), Sung Biographies, Wiesbaden, Franz Steiner Verlag, 1976, p. 613-615 ; cf. aussi à propos de son cuvre, « Sung Lin Ho-ching hsien-sheng shih-chi », in Étienne Balazs et Yves Hervouet (éds.), A Sung Bibliography (Bibliographie des Sung), Hong Kong, The Chinese University Press, p. 378.

13 Cité dans la Recette 10 et la Recette 68. Pour faciliter leur repérage dans le cadre de cette étude, les recettes ont été numérotées selon leur ordre d'apparition.

14 Cf. Shanjia qingshi, sect. Zhong mei yang he tuji, in Shuofu, Hanfenlou, 22/31b. 


\section{Françoise Sabban}

un autre de ses textes au titre proche de celui qui nous intéresse ici. $\mathrm{Ce}$ rappel n'est certes pas fortuit dans la mesure où, comme les titres de ses deux ouvrages le suggèrent, Lin Hong se place manifestement dans cette longue lignée d'« ermites laïques » qui existe en Chine depuis la fin des Han.

Cela dit, à part quelques confidences glissées entre les lignes sur son passage dans tel ou tel lieu, sa visite chez Untel, le souvenir d'un mets de Quanzhou, sa ville natale, nous ne savons pas grand-chose de la vie de Lin Hong. Il semble très cultivé, avoir beaucoup voyagé et rencontré du beau monde. A-t-il occupé un poste ou a-t-il suivi l'exemple de son illustre ancêtre Lin Bu, qui n'assuma jamais aucune charge officielle? Le $S J Q G$ comme le Shanjia qingshi laissent supposer que Lin Hong a été ou a aspiré à devenir un « lettré retiré " à l'instar de $\mathrm{Lin} \mathrm{Bu}$, son ancêtre ${ }^{15}$. En se qualifiant de shanjia ou « montagnard, homme de la montagne ", il s'assimilait à ces personnes qui avaient élu domicile dans les montagnes et les forêts à l'écart de la « civilisation », refusant ainsi les contraintes, les responsabilités, mais aussi le pouvoir que représentait l'investissement dans les affaires publiques. Contrairement à Lin Bu, Lin Hong a pris femme, puisqu'il fait brièvement allusion à la présence de son épouse comme cuisinière ${ }^{16}$.

Toute la difficulté consiste à savoir ce qu'on entendait exactement par «montagnes » et « forêts ». Ces lieux « hors du monde » étaient-ils aussi

15 Dans la Recette 23, il dit : " $p u$ ju shan shi » (quand j'habitais la montagne), ce qui suggère qu'il ne l'habite plus au moment où il écrit. Dans la Recette 34, après avoir évoqué ses voyages au Jiangxi, il mentionne son retour à la capitale, où chaque printemps lui fait penser aux armoises mangées autrefois au Jiangxi. Enfin, dans la Recette 71 , il déclare qu'après avoir voyagé pendant vingt automnes dans la région du Yangzi et de la Huaihe, à chaque saison des morts, il souhaite tout abandonner pour réaliser son grand souhait. La périphrase « tout abandonner » traduit l'expression fugui, une référence claire au célèbre $f u$ de Tao Yuanming Guiqu lai xi ci (Le retour) où, selon Wu Ke, l'éditeur récent du $S J Q G$, le poète exprime son désir de retourner chez lui après avoir abandonné son poste. Voir infra p. 32 et James Robert Hightower, « The $F u$ of T'ao Ch'ien », in John L. Bishop, Studies in Chinese Literature, Cambridge, Harvard University Press, 1965, p. 45-107 (« Harvard Yenching Institute Studies », 21).

16 Cf. Recette 74. 
sauvages que leur nom le laisse supposer ou bien la terminologie fondée sur le mot shan ne cachait-elle pas dans certains cas une convention? La question vaut d'être posée, car la «montagne » n'est pas simplement une référence bucolique ou rustique qui témoignerait d'une extravagance passagère. Le style de vie prôné par Lin Hong, s'il est fondé sur des critères de distinction qui tranchent avec les valeurs de la culture dominante, correspond en fait à une véritable pratique sociale reconnue. Nous reviendrons sur cette question et contentons-nous pour l'instant d'imaginer Lin Hong sous les traits d'un moral hero, ainsi qu'Alan Berkowitz ${ }^{17}$ appelle le lettré reclus chinois, une figure qui, par sa constance tout au long de l'histoire chinoise depuis les Han, constitue un fait social. Sans entrer dans le détail de ce phénomène dont les significations ont évolué ${ }^{18}$, rappelons que pour le héros moral «withdrawal usually meant withdrawal from active participation in an official career in the state bureaucracy. Reclusion was typically secular, and religious devotion was but one of any number of avocations pursued by individuals who had renounced public service », selon les termes d'A. Berkowitz ${ }^{19}$.

L'ouvre

Arrêtons-nous d'abord sur le titre de ce texte. Shanjia désigne l'homme qui vit en montagne au sens littéral du terme ${ }^{20}$. Le mot shan(lin) définit en effet un paradigme contenant toute une série de composés (shanren,

17 Cf. A.J. Berkowitz, "The Moral Hero. A Pattern of Reclusion in Traditional China » et « Reclusion in Traditional China. A Selected List of Bibliography », Monumenta Serica, 40, 1992, p. 1-32 et p. 33-46, ainsi que sa thèse « Patterns of Reclusion in Early and Early Medieval China. A Study of the Formulation of the Practice of Reclusion in China and its Portrayal " (Ph.D. Dissertation, University of Washington, 1989). Cf. aussi Donald Holzman, « Une conception chinoise du héros », Diogène, 36, 1961, p. 37-55.

$18 \mathrm{Cf}$. à ce sujet $\mathrm{Li}$ Chi, «The Changing Concept of the Recluse in Chinese Litterature», Harvard Journal of Asiatic Studies, 24, 1962-1963, p. 234-247.

19 Cf. A. Berkowitz, op. cit., 1992, p. 2.

20 Les compilateurs d'une bibliographie récente de textes culinaires chinois (Tao Zhengang, Zhang Lianming, Zhongguo pengren wenxian tiyao, p. 32) estiment 


\section{Françoise Sabban}

shanmin, shanfang, shanju, shanke, shanting, etc.) référant soit à l' ' homme de la montagne » soit à son lieu d'habitation. Lin Hong lui-même recourt plus de vingt fois à cette terminologie pour qualifier des mets (shanlin zhi $w e i$ ), des amis (shanlin pengyou), ou pour réaffirmer qu'il s'exprime du point de vue de ceux qui vivent en montagne, et dire que c'est ainsi qu'il convient de faire à la montagne.

Le sens de la deuxième partie du titre qinggong est moins clair, gong désigne la nourriture et les vivres, tandis que qing connote la pureté, la simplicité ${ }^{21}$, le naturel, la modestie, mais aussi l'élégance et le raffinement. Il est difficile de choisir parmi toutes ces acceptions, et il faut admettre l'ambiguité de ce terme qui, lorsqu'il est employé à propos de mets dans ce texte, évoque le plus souvent le raffinement et la simplicité, quoique dans certains contextes il se rapporte manifestement à la pureté bouddhique liée à l'interdiction d'atteinte à la vie ${ }^{22}$. Mais cela ne suffit pas à qualifier Lin Hong de bouddhiste. Il était probablement comme nombre de ses contemporains sensible à l'éthique bouddhiste, sans en être nécessairement un adepte. Pour ce qui est du composé qinggong, il pouvait aussi désigner les offrandes choisies de mets, d'encens ou de fleurs effectuées lors des fêtes calendaires. Le terme conserve ici l'idée de don, de cadeau ; à preuve, les nombreuses recettes de plats spécialement confectionnés pour des visiteurs. Toutefois qinggong désigne bien des produits alimentaires, car l'autre texte de Lin Hong au titre très proche, le Shanjia qingshi, traite de l'aménagement de la maison de montagne. C'est pourquoi Shinoda, le premier historien de l'alimentation chinoise, opérant un classement des différents types de textes culinaires, ouvre une catégorie qinggonglei ${ }^{23}$, voulant par là circonscrire un genre d'écrits dont les auteurs ont surtout porté leur attention sur la nature et ses produits. En effet, bien qu'il soit difficile de classer cet ouvrage

que le terme shanjia provient d'un poème de Du Fu. On trouve une citation de $\mathrm{Du} \mathrm{Fu}$, mais pas la même, toujours à propos de cette entrée dans le dictionnaire Ciyuan, vol. 2, Pékin, Shangwu yinshuguan, 1983, p. 920-921.

21 Pour Wang Xuetai (op. cit., 1993, p. 259), le terme signifie «nourritures bucoliques extrêmement raffinées" ".

22 Voir par exemple Recette 64.

23 Cf. Shinoda, op. cit., p. 147-154, 182-188, 209-212. 


\section{La diète parfaite d'un lettré sous les Song du Sud}

et d'interpréter son titre - ce texte n'ayant pas été retenu par les compilateurs du Siku -, il ne fait guère de doute qu'il s'intègre dans une tradition, celle de ces hommes qui, s'étant retirés de la vie publique, durablement ou momentanément, ont acquis une expérience qu'ils entendent parfois communiquer. Pour le spécialiste de l'histoire de l'agriculture $\mathrm{Hu}$ Daojing, ce mode de vie fait système, et le conduit à créer dans l'ensemble des traités d'agriculture une sous-classe dénommée shanju xitong ${ }^{24}$ « habitat montagnard $»$.

Le $S J Q G$ se compose de cent quatre courtes rubriques indépendantes, dont le thème est un mets, un produit ou une plante alimentaires. Leur succession ne paraît obéir à aucun ordre particulier, contrairement aux traités culinaires qui sont en principe structurés par genres de plats ou d'ingrédients. Chacun de ces textes est individualisé par un titre qui, le plus souvent, ne peut se comprendre qu'après la lecture du passage dans son entier. Quant à ceux qui paraissent transparents, à quelques exceptions près, ils prêtent à confusion si l'on se contente d'en déchiffrer le sens littéral. Toutes les astuces de la rhétorique et les ressources de sa culture sont ainși convoquées par Lin Hong pour intituler ces brefs discours, comme s'il avait voulu d'emblée susciter la curiosité. Un potage clair à l'âche d'eau ${ }^{25}$ est appelé Potage de la ravine verte (Recette 2 ) d'après un vers de $\mathrm{Du} F u$; les Feuilles de jade croustillantes (Recette 28 ) sont des toasts chauds enduits de miel ou de beurre qui craquent sous la dent comme le crissement des pas sur la neige que Yang Wanli fait entendre dans l'un de ses poèmes ; les Chaussons mermontagne (Recette 37$)^{26}$ désignent de manière plaisante de petits rouleaux

24 Sous-classe inaugurée selon lui par le Shanju yaoshu (Techniques essentielles pour la résidence en montagne) de Wang Min des Tang. Cf. Hu Daojing, Nongshu. Nongshi lunji (Traités d'agriculture. Essais sur l'histoire de l'agriculture), Pékin, Nongye chubanshe, 1985 p. 30-31. Cf. aussi Wang Yuhu, Zhongguo nongxue shulu (Bibliographie agronomique chinoise), Pékin, Nongye chubanshe, 1964, p. 39.

25 Apium graveolens $\mathrm{L}$.

26 Cette désignation est d'autant plus amusante que le poisson et les crevettes entrant dans la farce de ces chaussons, et dont les dénominations ne comportent aucune spécification qui pourraient faire penser le contraire (simplement yu et $x i a$ ), provenaient très certainement d'une pêche d'eau douce. Mais il s'agit ici 


\section{Françoise Sabban}

farcis de fruits de mer, de pousses de bambou et de crosses de fougère ; $L a$ montagne embaumée (Recette 74) est la morale concise d'une histoire qu'il faut suivre de bout en bout pour comprendre que l'on traite dans cette recette d'un mélange d'épices spécialement destiné à la préparation des légumes. Le titre est une énigme qui trouve à la fin sa solution comme une intrigue son dénouement. Et Lin Hong de souligner, en forme de pléonasme parfois, que telle est la raison qui fonde la dénomination.

En outre, chaque recette, unique par son contenu, l'est aussi dans sa forme. Les énoncés formulaires propres aux traités techniques sont assez peu présents ; celui du Caillé de soja à la (Su) Dongpo (Recette 82), dont le texte tient dans la seule énumération d'opérations culinaires de base : « Faire revenir le caillé dans de l'huile avec de la ciboule, puis poursuivre la cuisson avec de la sauce jiang ${ }^{27}$ mélangée à une dizaine ou une vingtaine de graines de torreya ${ }^{28}$ pilées », en est l'un des rares exemples. Fréquemment, Lin Hong maintient la tension narrative posée par des titres énigmatiques en faisant de la plupart de ses recettes des anecdotes, bonnes à raconter. Mais il s'agit d'anecdotes de haute tenue, où la poésie et l'humour se marient à la curiosité scientifique, voire ethnologique de l'auteur, à ses souvenirs et aux nombreux témoignages de sa culture.

Ainsi Lin Hong, partant de l'alimentaire, conduit-il son lecteur en des lieux divers : des joies du fourneau aux plaisirs du jardinage, de la nostalgie du passé aux dernières modes, des leçons de l'histoire à la vanité des choses, des techniques anciennes ou modernes à la philosophie de l'existence. Les motifs du paysage parcouru sont innombrables. Veut-on s'initier à la confection des Pâtes au bulbe de lys ${ }^{29}$ (Recette 17) que l'on apprend comment du rhizome récolté au printemps ou à l'automne on extrait une fécule apte à

de se conformer à un cliché poétique classique qui oppose nécessairement la mer à la montagne dans leurs productions : pour la montagne les plantes de la forêt, pour la mer ce qui vit dans l'eau.

27 Jiang, condiment pâteux fermenté, à base de céréales et/ou de légumineuses, dont on utilisait soit la partie consistante, soit le liquide résiduel (de là, sauce de soja jiangyou).

28 Torreya grandis Fort.

29 Lilium brownii F.E. Brown. 
être transformée en pâtes alimentaires spécialement bonnes pour le souffle et le sang ; mais aussi à quelle date il faut le planter, et pourquoi il faut l'engraisser avec de la fiente de poulet, seule fumure qui, toujours selon Lin Hong, favorise la mutation des vers de terre en bulbes. La leçon, parfois moins savante, est frappée au coin du bon sens : la description de la préparation de la moelle de laitue en vinaigrette dans la recette de la Laitueasperge croquante ${ }^{30}$ (Recette 89 ) est l'occasion de rappeler le triste sort de $\mathrm{Du} \mathrm{Fu}$, qui, échouant à faire lever ses semis, constate avec amertume que «le chemin de la vie n'est pas une route plane».

On voit que le $S J Q G$ est un ouvrage inclassable, qui tient du manuel de cuisine ou de philosophie quotidienne, de l'encyclopédie domestique ou du traité d'agriculture pratique, du florilège ou de la confession d'un moraliste. Si le contenu proprement culinaire en fait un livre de cuisine, la forme, élaborée par les références classiques ${ }^{31}$, les citations, les jeux de mots relèvent d'un autre genre ${ }^{32}$. Mais le $S J Q G$ n'est pas seulement l'œuvre brillante d'une belle plume. Son originalité réside dans l'objectif de son auteur, un homme de conviction désireux de faire partager sa conception du monde et ses inclinations esthétiques. Or, Lin Hong a fait le choix du retrait dans les montagnes - à moins qu'il ne s'exprime au nom de ceux qui ont fait ce choix - , ce qui ne peut manquer d'avoir une incidence sur

30 Lactuca sativa $L$.

31 Lin Hong truffe son texte de citations d'illustres poètes des temps passés, au rang desquels Du Fu des Tang et Su Dongpo des Song du Nord sont ses préférés, des extraits de leurs œuvres étant mentionnés plus de dix fois. Mais ni Li Bai, ni Tao Yuanming, ni surtout Yang Wanli ou Huang Tingjian ne sont oubliés. D'autres moins connus sont aussi cités à l'occasion, ainsi que Zhu Xi ou certains grands personnages historiques, fondateurs de dynasties, empereurs, ou généraux dont les noms et les hauts faits ont été conservés dans les Histoires dynastiques.

32 L'esprit ludique de Lin Hong n'est pas sans rappeler le Qingyilu (Récits étranges), un recueil de savoureuses anecdotes de l'époque des Tang et des Cinq Dynasties attribué à Tao Gu (903-970), où, dans la section sur le boire et la manger, chaque histoire tournant autour d'un plat ou d'un aliment est tout entière symbolisée par un titre plein d'esprit. À propos de cet ouvrage, cf. Étienne Balazs et Yves Hervouet (éds.), A Sung Bibliography, p. 320. 


\section{Françoise Sabban}

la conduite de l'existence. L'éloignement du monde des affaires a pour corollaire un rapprochement avec la nature qui devient un lieu de vie, une source d'inspiration et plus prosaïquement celle de l'approvisionnement.

\section{La cuisine et la gastronomie du montagnard}

\section{Une exploitation des ressources du milieu}

Il faut en effet supposer que l'environnement proche ou éloigné du studio de Lin Hong lui fournit la plupart des ingrédients de ses recettes, puisque la grosse majorité d'entre elles concerne une ou plusieurs plantes alimentaires. En effet, plus d'une soixantaine d'espèces différentes, auxquelles il faut ajouter un certain nombre d'herbes aromatiques, sont utilisées. Ces plantes font bien partie des codex pharmaceutiques chinois, mais elles n'appartiennent pas toutes au registre de la cuisine ordinaire. Si l'on retrouve le chou chinois, le haricot d'Égypte, la laitue-asperge, les pousses de soja, les tiges de zizanie, la ciboule chinoise, les pousses de bambou, la petite rave, les haricots rouges, la courge calebasse, les aubergines $^{33}$, etc., tous légumes choisis mais relativement courants selon les régions, on relève aussi quantité de racines, de pousses, de boutons, de fleurs et de graines de plantes, habituellement absents des traités culinaires. Là réside probablement l'une des originalités de ce texte, dans la mesure où la cuisine de ces végétaux plus ou moins sauvages est intégrée dans une pratique culinaire domestique. Ainsi, à son échelle, le $S J Q G$ préfigure l' "esculentist movement " des Ming, selon la terminologie de Joseph Needham, caractérisé par la publication d'une série d'herbiers, et en particulier d'herbiers de famine contenant une grande variété de plantes de cueillette et dont les auteurs préconisent l'exploitation culinaire ${ }^{34}$.

33 Brassica chinensis L., Dolichos lablab L., Glycine max (L.) Merr., Zizania caduciflora (Turcz.) Hand.-Mazz., Allium fistulosum L., Phyllostachys pubescens Mazel. Ex H., Raphanus sativus L., Phaseolus calcaratus Roxb., Lagenaria siceraria (Molina) Standl. var. gourda Ser., Solanum melongena L. $34 \mathrm{Cf}$. à ce propos le plus célèbre de ces manuels et le premier d'entre eux, le 
Si la nature dans son domaine végétal est largement exploitée, le monde animal est peu représenté, mais les espèces sélectionnées en sont particulières ; il s'agit pour les animaux vivant sur terre surtout de gibiers : cerfs hydropotes, lièvres, canards mandarins et chats sauvages ${ }^{35}$, mais aussi plus prosaïquement poulets et moutons. Pour les espèces aquatiques, on relève le poisson sans autre précision, le poisson séché ou le poissonmandarin ${ }^{36}$, les crevettes et surtout les crabes d'eau douce ${ }^{37}$, vivement appréciés, semble-t-il.

À cet approvisionnement local il faut ajouter quelques produits manufacturés, le caillé de soja ou le gluten, typiques de la cuisine végétarienne ${ }^{38}$, ainsi que les adjuvants nécessaires à toute véritable activité culinaire, le sel, l'huile, le sucre, le miel, le vinaigre, la sauce jiang, la sauce de soja, le ferment hongqu utilisé comme colorant rouge, la farine et la fécule ${ }^{39}$. En somme, on relève très peu d'ingrédients qui ne soient directement tirés du milieu naturel proche; le vin ${ }^{40}$ même pouvait être confectionné par les soins du lettré retiré, puisque Lin Hong consacre un

Jiuhuang bencao (Materia medica de secours contre la famine), écrit en 1406 par Zhu Xiao, le cinquième fils du fondateur de la dynastie des Ming. Cf. Amano Motonosuke, « Mindaini okeru Kyûkô Sakubutsu Chojutsukô », Tôyô Gakuhô, 47.1, 1964, p. 32-59. Cf. Aussi J. Needham, Lu Gwei-Djen et Huang Hsing-Tsung, Science and Civilisation in China, Vol. 6 : Biology and Biological Technology, Part $1:$ Botany, p. 328-355, ainsi que J. Needham et Lu GweiDjen, « The Esculentist Movement in Mediaeval Chinese Botany. Studies on Wild Emergency Food Plants ", Archives Internationales d'Histoire des Sciences, 21, 1968, p. 225-248.

35 Hydropotes inermis Swinhoe, Lepus tolai Pallas (?), Aix galericulata (L.), Felis bengalensis Kerr.

36 Siniperca chuatsi (Basilewsky).

37 Eriocheir sinensis $\mathrm{H}$. Milne-Edwards, Macrobrachium nipponense (de Haan).

38 Je regroupe sous cet intitulé des recettes comportant dans leur titre le mot $s u$, ainsi que quelques autres, comme nous le verrons infra en p. 35-40.

39 Il faut ajouter à cette liste les feuilles de pâte de haricot mungo qui servent d'enveloppes aux Chaussons mer-montagne (Recette 37), ainsi que le poisson séché (Recette 79), considéré dans ce texte comme une nourriture de voyage et comme un ingrédient de la cuisine.

40 Le mot « vin » traduit ici le terme chinois $j i u$ et désigne une boisson alcoolique issue de la fermentation d'un moût de céréale, titrant de $10^{\circ}$ à $18^{\circ}$. 


\section{Françoise Sabban}

long développement en fin d'ouvrage à cette fabrication difficile. Néanmoins, la présence de condiments de base, qui donnent toute leur saveur à la cuisine, montre que le montagnard n'était nullement coupé du commerce alimentaire ordinaire et que, s'il choisissait de consommer et de préparer un certain type de cuisine, comme nous allons le voir, il ne pouvait se passer des media indispensables qui font d'une préparation comestible autre chose qu'un simple trompe-la-faim.

\section{Répertoire des plats}

Le SJQG concerne la cuisine de l'immédiat, celle que l'on mange dans l'instant ou dans un bref laps de temps après sa confection ; de ce fait, il contient extrêmement peu de recettes de mets destinés à la conservation ${ }^{41}$ ou nécessitant une consommation différée ${ }^{42}$. Lin Hong dresse d'ailleurs un inventaire complet des types de plats du répertoire culinaire, mais sans aucun ordre repérable. Ainsi sont représentés les mets constitutifs du menu chinois, de même que les en-cas, typiques des prises alimentaires hors repas : les préparations céréalières (en grains entiers, en porridges ou bouillies, sous forme de pâtes alimentaires), éléments substantiels, côtoient les nombreux plats d'accompagnement, essentiellement à base de légumes, ainsi que les nourritures de plaisir que sont galettes, beignets, gâteaux et autres confiseries.

La plus grosse part des recettes, un tiers environ, sont des plats de «légumes» confectionnés avec une ou plusieurs plantes associées,

41. Les exceptions à cette règle sont : une « choucroute » (Recette 98 ), une conserve de racine de bardane (Recette 96), une confiture d'ophiopogon à manger de suite ou à garder (Recette 63) et certaines préparations complexes de produits dérivés d'une plante comme le caillé de lait de pavot (Recette 84), issu de la transformation des graines de pavot en un lait qui sera caillé, puis mis en forme et découpé en petits morceaux pour être cuisinés par la suite ; ou encore les fécules de châtaignes d'eau (Heleocharis dulcis (Burm. F.) Trin. ex Henschel, Recette 32), d'ignames (Dioscorea opposita Thunb., Recette 66) ou d'anguine (Trichosanthes kirilowii Maxim., Recette 18), dont les longues procédures de fabrication sont rapidement décrites, et qui serviront ultérieurement comme agent de liaison ou comme ingrédient de base de vermicelles.

42 Ce qui est souvent le cas dans les traités culinaires chinois. 
rapidement cuites, puis assaisonnées. Viennent ensuite les mets céréaliers qui, fait remarquable, sont toujours cuits avec un autre élément végétal, fleur ou feuille : il en est ainsi des préparations en grains séparés et des porridges plus aqueux, mais aussi de certaines pâtes alimentaires fraîches ${ }^{43}$, à base d'un mélange de farine de blé ou de fécule et de jus de feuilles, de racines ou de tiges. Les autres recettes, en-cas ou plats d'accompagnement de viande ou de poisson, sont beaucoup moins nombreuses. Elles contiennent cependant toujours des plantes utilisées pour leurs propriétés aromatiques ou médicinales. Notons enfin qu'on ne relève que trois mentions de bouillon ou jus de viande, servant à la confection de potages ${ }^{44}$. Les autres sont en effet tous à base de céréales et de légumes, ce qui constitue une spécificité du traité de Lin Hong, car les bouillons de viande serviront de fonds de cuisine à une grande quantité de plats dans les traités ultérieurs sous les Yuan.

\section{Une cuisine inventive et de haut goût}

Malgré l'intérêt que Lin Hong porte à la flore, dont on pourrait penser qu'il veuille en consommer les espèces au plus près de leur état naturel, sa cuisine accorde une place négligeable au cru. La gastronomie de Lin Hong est une gastronomie du cuit. Ne font en effet exception à cette règle que des fruits : des poires arrosées ${ }^{45}$ de jus d'orange ${ }^{46}$, de sel et de sauce jiang (Recette 65), ainsi que des châtaignes ${ }^{47}$ et des olives chinoises ${ }^{48}$ dont les

43 Cf. à ce propos F. Sabban-Serventi, « Ravioli cristallins et tagliatelle rouges. Les pâtes chinoises entre XII et XIve siècle », Médiévales, 16-17, 1989, p. 2950 («Plantes, mets et mots. Dialogues avec André Georges Haudricourt »).

44 Un bouillon de poulet pour servir les Raviolis fleurs de prunus (Recette 14), un coulis, toujours de poulet, ajouté aux Perles de grenade (Recette 77) et un jus de mouton pour allonger le Potage d'or et de jade (Recette 94) à base de châtaignes et d'ignames.

45 Pyrus bretschneideri Rahd.

46 Citrus junos Tanaka.

47 Shanli, litt. «châtaigne de montagne »; je n'ai pas trouvé l'identification botanique de cette plante pour l'instant.

48 Canarium album (Lour.) Raeusch. 
fines tranches sont elles aussi saupoudrées de sel (Recette 92). Cette prédilection pour le cuit ne surprend pas aujourd'hui, la cuisine chinoise ne laissant quasiment aucune place à des préparations crues. Ce n'était pas le cas sous les Song, ni ultérieurement sous les Yuan, et cela n'avait pas été le cas auparavant. Lin Hong fait d'ailleurs allusion de façon métaphorique à l'un des plats les plus célèbres de la gastronomie chinoise ancienne, dénommé kuai, et qui était en fait un sashimi à la chinoise, un plat de poisson cru très apprécié, qu'il appelle Fils d'argent, une dénomination laudative connotant les cordes d'un luth ${ }^{49}$ (Recette 31 ), mais aucune recette concrète de kuai n'est donnée dans le texte.

Cependant, la cuisson dans le $S J Q G$ procède d'un art bien maîtrisé. Bien que les cuissons en milieu humide prédominent largement sur le grillé, le rôti ou le frit, leur élaboration n'en est pas moins raffinée. Ainsi, les végétaux, cueillis le plus souvent très jeunes, et qui sont donc très tendres, sont à peine ébouillantés avant d'être assaisonnés. Comme par exemple les jets d'armoise, les petites feuilles de lampourde ou les bourgeons de bambou, de lyciet, et les tiges naissantes de zizanie ${ }^{50}$. Et lorsqu'ils sont cuits en potage (geng), on les poche aussi de manière à ne jamais trop les cuire, ne serait-ce que pour conserver leur couleur verte ${ }^{51}$. Accommodés avec une céréale, ces éléments végétaux, qu'il s'agisse de sommités florales ou de feuilles, ne sont ajoutés qu'en cours de processus, la céréale nécessitant un temps de cuisson plus long ${ }^{52}$. Le « sauté à la chinoise » (chao*), aujourd'hui mode universel de préparation des plats quotidiens, et en particulier des légumes, n'est employé que dans deux ou trois recettes, et l'identification

49 Voir infra note 65 et texte correspondant.

50 Artemisia vulgaris L., Xantium sibiricum Patr. ex Widd., Lycium chinense Mill., Zizania caduciflora (Turcz.) Hand.-Mazz. (Recettes 34, 36, 55).

51 C'est le cas de l'âche d'eau dans la Recette 2, des pousses de chrysanthème (Recette 30) ou encore des crosses de fougère (Recette 37), etc.

52 Cf. par exemple la Recette 52 : « Cueillir de véritables chrysanthèmes de couleur jaune à la tige violette. Les ébouillanter rapidement dans une décoction de réglisse. Attendre que le riz soit à moitié cuit, puis les ajouter, et faire cuire ensemble. » 
n'en est pas sûre, car le verbe spécifique moderne n'apparait que deux fois $^{53}$. Malgré le petit nombre de recettes de viande, Lin Hong a choisi des modes de cuisson appropriés aux types de chairs cuisinées : deux ragoûts, un de poulet à l'huile de sésame (Recette 11) et l'autre de mouton aux amandes (Recette 95); une fondue de lièvre (Recette 38); une galantine de chat sauvage entier découpé en tranches fines pour le service (Recette 93 ) ; deux grillades de gibiers : un couple de canards mandarins enduits d'huile et rôti à la flamme avec assaisonnements (Recette 68) et une pièce de cerf marinée dans du vin et des épices, puis grillée à feu vif, protégée par une couche de graisse de mouton (Recette 90). On voit que Lin Hong ne dédaigne pas du tout le rôti, cuisson adaptée au jeune gibier à plume et à la venaison qui est ici savamment enrobée dans de la graisse pour nourrir la chair et éviter qu'elle ne se dessèche à la chaleur directe du feu. Cette technique de cuisson peu employée, pour ne pas dire inconnue aujourd'hui dans la sphère domestique, paraît sous le pinceau de Lin Hong parfaitement appropriée. Ces préparations entre cuisine à la maison et cuisine de chasse symbolisent les choix du montagnard qui, à cheval entre deux sociétés, peut aisément tirer parti de leurs avantages réciproques. La cuisson des poissons et des crabes, de même, est bien pensée. Le crabe est cuit sans excès avec de l'orange ou du vinaigre, de l'âche d'eau et, à la rigueur, de la ciboule (Recettes 44 et 49 ); le poisson-mandarin, étuvé dans un réceptacle de graines de lotus avec du vin et des condiments (Recette 45). Cela dit, les techniques culinaires déployées par Lin Hong sont, dans l'ensemble, assez simples. On ne relève que très peu de cuissons mixtes ou de longues opérations de précuissons si caractéristiques aujourd'hui de la grande cuisine chinoise ${ }^{54}$.

L'assaisonnement des plats est modulé avec une extrême précision. Lin Hong travaille avec une gamme ascendante des saveurs, qui tient compte

53 Il n'y a guère que les pousses des légumineuses (Recette 29) et les pousses de chrysanthème (Recette 30 ) dont nous pouvons affirmer qu'elles sont « sautées ». Cela n'est que probable pour les pousses de soja (Recette 71), qui sont bien « cuites » dans l'huile selon le mode de cuisson désigné par le verbe homophone chao, dont la signification ne nous est pas parfaitement claire.

54 Cf. F. Sabban, «Le système des cuissons dans la tradition culinaire chinoise », Annales. Économies. Sociétés. Civilisations, 2, 1983, p. 341-368. 
de la nature de l'ingrédient. Toutes les expériences gustatives, de la quasifadeur à la puissance aromatique intense, sont ainsi explorées. Dans le registre des saveurs minimales, quoi de plus subtil que le Potage de pierres blanches (Recette 53), résultat de l'ébullition prolongée de petits cailloux blancs rongés de lichen dans de l'eau de source ? Lin Hong parle d'une «saveur plus douce que celle de l'escargot " et d'un «parfum de pierres de fontaine ${ }^{55}$. Le Porridge auxfleurs de prunus n'est pas moins recherché dans l'extrême simplicité de son goût : "Ramasser des fleurs de prunus et bien les laver. Faire cuire du riz blanc de première qualité dans de l'eau de neige fondue. Quand il est cuit, ajouter les fleurs et donner un bouillon » (Recette 54). Comme le porridge aux fleurs de prunus, des beignets, des petits gâteaux, des confiseries sont aromatisés sans excès par des essences végétales. Les Palets de Dongting (Recette 57), cadeaux du moine Jingju au philosophe Ye Shi (1150-1223), ne sont pas plus gros qu'une sapèque, mais leur frais parfum évoque les orangeraies de Dongting. Interrogé sur le secret de leur arôme, le moine explique que chaque petit gâteau a été enveloppé dans une feuille d'oranger avant d'être cuit à la vapeur ${ }^{56}$. Lin Hong semble avoir une prédilection pour ces parfums qui se transmuent en saveurs, ces bouffées délicieuses qui se dégustent au nez et dans la bouche. Les pétales de pivoine ou de gardénia exhalent ainsi une double senteur lorsque, enrobés d'un léger nuage de farine, ils deviennent dans la friture de croustillants beignets (Recettes 97 et 33). Cette utilisation des terminaisons florales ou des jeunes pousses à l'arôme à peine confirmé pour donner un goût unique, souvent léger, est probablement l'une des spécificités

55 L'idée d'un « potage de cailloux » n'est pas propre aux Chinois. Dans un texte consacré aux plats pauvres de la cuisine livournaise, d'une époque aujourd'hui révolue, il est fait mention d'une "minestrina di sassi ", ou consommé de cailloux, obtenu à partir de l'ébullition de pierres spongieuses, typiques des fonds marins de la région. Cf. Giovanni Petagna,. Brodo di sassi. Curiosità gastronomiche nella storia livornese, Livourne, Editrice Nuova Fortezza, 1966, p. 11-13.

56 Les oranges de Dongting, du nom des collines situées sur une île et une presqu'île du Lac Taihu au Jiangsu étaient très réputées pour leur parfum, la finesse de leur peau et leur précocité. 
de cet ouvrage, et témoigne véritablement d'un choix personnel et original de Lin Hong, qui semble en l'occurrence s'appuyer sur ses propres expériences ou celles de ses proches. Mais il sait aussi se conformer au modèle canonique de l'assaisonnement chinois et à ses techniques. Il fait ainsi appel à une riche palette d'ingrédients donnant du goût, mêlant les condiments manufacturés (vinaigre, sauce jiang, sauce de soja ${ }^{57}$, marc de vin, vin, purée de sésame, sucre) aux plantes aromatiques classiques du répertoire chinois (gingembre, clavalier, poivre, anis, aneth, zeste et pulpe d'orange, ciboule ${ }^{58}$ ). Lin Hong ne se contente pas d'ajouter quelque sauce par-ci par-là, il dispense les adjuvants aromatiques avec le savoir-faire que confèrent l'habitude et le métier. Il est un moment pour assaisonner, comme il est un moment pour parachever une cuisson. Le Poulet jaune d'or est poché dans un court-bouillon parfumé à l'huile de sésame, à la ciboule et au clavalier. Le jus, réduit après cuisson, est reversé sur le poulet découpé pour que la chair s'imprègne à nouveau de la concentration des saveurs (Recette 11). Effet inverse, en quelque sorte, dans L'offrande de nuées empourprées (Recette 38), c'est avant d'être pochée dans un bouillon que la chair du lièvre, tranchée en fines lamelles, est mise à mariner dans un

57 Il est probable que la sauce jiang et la sauce de soja ne soient, encore à l'époque, qu'un seul et même produit, le second n'étant que la partie liquide du premier (cf. à ce propos F. Sabban, «Insights into the Problems of Preservation by Fermentation in 6th Century China », in Astri Riddervold et Andreas Ropeid, Food Conservation. Ethnological Studies, Londres, Prospect Books, 1988, p. 52, note 12, et ci-dessus, note 27); n'ayant pu éclaircir cette question pour l'instant, je les compte comme deux produits distincts.

58 À cette liste il faut ajouter des épices non spécifiées, appelées xiangliao ou liaowu. Il est difficile de dire à quoi renvoient ces termes. Il n'est pas sûr que liaowu signifie déjà «mélange d'épices » au sens de "mélange d'épices orientales » prêt à l'emploi, comme cela sera le cas ultérieurement sous les Yuan (cf. F. Sabban, « Court Cuisine in Fourteenth-Century Imperial China. Some Culinary Aspects of Hu Sihui's Yinshan zhengyao », Food \& Foodways, $1.2,1986$, p. 177), dans la mesure où le mélange d'épices à conserver de la Recette 74 n'est pas du tout désigné par ce terme. Par ailleurs, dans la Recette 93, le même terme désigne clairement l'ensemble des aromates énumérés précédemment dans le corps du texte, et non pas une préparation particulière. 
mélange de vin, de sauce jiang et d'épices poivrées. Nul doute que la fondue de lièvre aura le goût recherché et que la viande ne sera nullement délavée par sa cuisson. On perçoit dans le $S J Q G$ une histoire déjà ancienne des complexes aromatiques liés à tel ou tel aliment : ainsi, le poisson ne peut se cuisiner sans le vin, de même que le crabe est nécessairement associé avec l'orange et le vinaigre ${ }^{59}$, tandis que les légumes ont besoin au minimum de la force de la sauce jiang ou du piquant du gingembre pour mettre en valeur leur propre saveur. En vérité tous les plats que l'on qualifierait aujourd'hui de $c a i^{60}$ et qui sont considérés comme les accompagnements d'une céréale, qu'ils soient à base de légumes, de viandes, de poissons ou d'autres produits, et quel que soit leur mode de cuisson, incluent un ou plusieurs des éléments aromatiques cités ci-dessus. La cuisine de Lin Hong est une cuisine de haut goût.

Mais l'une des originalités de Lin Hong, qui relève autant de l'exploitation intelligente du milieu naturel que du génie créatif d'un grand cuisinier, est l'usage détourné qu'il fait de certains éléments végétaux, soit qu'il les transforme en ustensiles culinaires tenant lieu de contenants décoratifs tout en tirant parti de leur saveur naturelle, soit qu'il les façonne pour créer une illusion visuelle et gustative. Il fait ainsi cuire le corail d'un crabe au cœur d'une orange en partie évidée de sa pulpe (Recette 44); il recycle le réceptacle à graines d'un lotus en logeant dans sa cavité un poisson-mandarin qui sera ensuite étuvé à la vapeur (Recette 45); il rapporte l'idée de Xie Yizhai qui fit ciseler deux coupes dans un cédrat ${ }^{61}$ (Recette 43 ) pour offrir

59 On peut lui ajouter d'autres aromates, mais il est d'emblée automatiquement associé à l'orange et au vinaigre. Cf. Recette 49 , où Lin Hong dit : « Ce produit est harmonieux, il suffit de vinaigre et d'orange pour exalter ce qu'il renferme. »

60 Ainsi que Lin Hong l'explique dans la Recette 47, le mot cai, à son époque, ne désigne pas encore les plats d'accompagnement, et signifie le plus souvent « légume».

61 Rien d'étonnant à ce que le grand restaurant de Hangzhou, Bagualou, aujourd'hui spécialisé dans la reconstitution de menus des Song, ait intégré ces dernières recettes à son répertoire. Voir les recettes réinterprétées et les photos les accompagnant dans Xu Hairong et al., Zhongguo Hangzhou Bagualou fang Songcai (Mets inspirés des Song. Restaurant Bagualou, Hangzhou, Chine), Pékin, Zhongguo shipin chubanshe, 1988, p. 6-9, 42-45. 
à boire à ses amis $!^{162}$ L'imagination du cuisinier aurait moins d'impact si elle n'était relayée par la fantaisie poétique pour dénommer ces inventions toutes de grâce et de raffinement : Crabe en orange farcie, Papillote de poisson en coupe de lotus, Des cédrats pour coupes à boire. La recette Perles de grenade (Recette 77 ) procède d'une inspiration comparable. Jouant sur les mots et les matières, ce plat est un délicieux mirage où l'art de Lin Hong atteint son apogée. Ici point de véritables graines de grenade malgré le nom, mais un faux-semblant de perles roses translucides nageant dans un bouillon légèrement lié, et qui cache un travail phénoménal : «Couper un rhizome de lotus en petits dés, les arrondir un à un en les polissant sur le fond rugueux d'un récipient en grès; les colorer avec une décoction de fleurs de prunus de Chine et de teinture rouge ${ }^{63}$; mélanger avec de la fécule de haricot mungo ${ }^{64}$, ajouter du coulis de poulet et faire cuire. On dirait des graines de grenade. »

\section{Les fondements théoriques de la cuisine de Lin Hong}

La sophistication de la cuisine du $S J Q G$ ne doit rien au hasard, elle est fondée sur une gastronomie, elle-même gouvernée par une théorie implicite des saveurs. Il s'agit moins du système traditionnel des cinq saveurs et de leurs correspondances que d'une conception propre à Lin Hong. L'auteur du $S J Q G$ recherche la saveur véritable et il s'inquiète souvent de la perdre ou de la voir trahie. Une parabole éclaire le lecteur. Dans la « recette » Fils $d$ 'argent (Recette 31), le fonctionnaire Zhang Yuezhai se moque gentiment de ses amis en ordonnant à ses gens qu'on prépare un plat de « fils d'argent correctement accommodés et ayant une saveur véritable ». Ses invités,

62 L'usage de contenants périssables existe toujours dans la cuisine chinoise populaire et gastronomique ; en Chine du Sud, de gros nœuds de bambou frais font d'excellents récipients pour la cuisson du riz; dans les banquets, il n'est pas rare qu'une soupe ou une salade de fruits soient présentées dans de grosses courges ou pastèques admirablement sculptées.

63 Yanzhi, nom d'un produit colorant en rouge utilisé en cosmétique, dont on relève l'usage dans les textes dès le vi ${ }^{e}$ siècle. Peut-être Basella rubra $\mathrm{L}$. ?

64 Phaseolus radiatus $\mathrm{L}$. 
obnubilés par leur gourmandise et augurant à l'avance le plaisir de la dégustation, s'écrient à l'unisson : «C'est, à n'en pas douter, une julienne de poisson cru en salade. » Zhang ne dit mot, mais se saisissant d'un luth prie le maître de musique de jouer un air du Lisao. Les " fils d'argent » ${ }^{65}$ n'étaient rien d'autre que les cordes du luth et « accommoder correctement » signifiait « accorder l'instrument». Quant à la saveur véritable, c'était la musique que Tao Yuanming tirait de son luth. Par cette métaphore qui assimile l'activité culinaire à celle du musicien, Lin Hong suggère que la saveur véritable d'un aliment s'actualise grâce à l'action du cuisinier qui, tel un musicien, ne produit des sons harmonieux que lorsqu'il est maître de son instrument. Il revient donc au cuisinier de révéler les saveurs véritables dans leur plénitude, à condition qu'il n'appartienne pas à cette confrérie de piètres maîtres queux tant redoutés par Lin Hong, et qui mettent par exemple tout leur zèle à assaisonner le crabe, le gâchant ainsi irrémédiablement (Recette 49). L'auteur du SJQG consent à reconnaître quelque talent à certains de ces gâte-sauce, mais il ne comprend pas pourquoi ils sont toujours si prompts à suivre les dernières modes, comme de poêler la volaille au lieu de la faire cuire au court-bouillon (Recette 11) ? « Ce n'est pas que nous les montagnards méprisions ces façons, dit Lin Hong, mais nous craignons que la saveur véritable ne soit perdue. »

Si le cuisinier doit faire montre de son talent de technicien pour ne pas décevoir, il doit en outre s'initier à la cuisine de montagne qui offre de véritables spécialités. Quelques plats sont honorés du label de qualité « mets typiques des montagnes et des forêts » (shanlin zhi wei): les Pâtes vertes (Recette 12), des tagliatelles teintées avec le jus de jeunes feuilles de sophora ; L'or rôti et le jade bouilli (Recette 24 ), des pousses de bambou en deux façons, des frites et croquantes ou des moelleuses mitonnées dans un porridge de riz; L'offrande de nuées empourprées, une fondue de lièvre dont le bouillon ressemble à un banc de nuages rougeoyants (Recette 38$)^{66}$;

65 L'expression " fils d'argent » est ici une double métaphore : elle connote le sashimi de poisson découpé en filaments dont l'éclat lumineux est comparable à l'argent, mais aussi les cordes brillantes du luth.

66 C'est par cette comparaison que j'interprète ce titre tiré d'un vers de Lin Hong, et cité dans le cours de son texte. 
les Champignons de jade en meurette (Recette 75), des champignons sauvages, ébouillantés puis pochés au vin. D'autres mets sont recommandés au montagnard ou lui conviennent particulièrement : il ne peut se passer du Porridge aux haricots (Recette 8), dans lequel les légumineuses longuement cuites ont consistance de beurre; il honore les Raviolis fleurs de prunus (Recette 14) en souvenir d'un lettré reclus du temps passé ; les Pilules de taro (Recette 25), en fait du taro cuit à l'étouffée sous la braise, est le mets idéal des réunions autour d'un feu de camp en pleine montagne ; les Galettes aux bourgeons de sapin (Recette 27), à base de miel étuvé aux senteurs de résineux, lui sont une source d'inspiration et de joie ; et au printemps, dans les « académies de montagne » du Jiangxi (Jiangxi lin shanfang shuyuan), on se régale d'Armoises en légume (Recette 34), etc.

Difficile de savoir quels sont les critères de la « typicité montagnarde » de ces plats. Leurs composants jouent certes un rôle : champignons sauvages, pousses de bambou, taro, pin, lièvres, sont les hôtes naturels des bois et des montagnes, et voir ainsi qualifier le plat où ils tiennent une place essentielle n'est pas surprenant. Mais il est bien d'autres recettes du texte dont l'ingrédient principal est un élément naturel sauvage et qui n'ont pas pour autant reçu cette marque de qualité. Ainsi, par exemple, est-ce leur couleur, le recours aux feuilles de sophora comme agent colorant ou encore leur valeur saisonnière qui concourent à distinguer les Pâtes vertes (Recette 12) ? Le sophora, arbre commun en Chine, exploité en pharmacopée et en teinture, n'appartient pas au registre culinaire ordinaire. L'utilisation peu courante des jeunes feuilles de cet arbre pourrait justifier le label. Mais dans la mesure où Lin Hong, en reproduisant un poème de Du Fu décrivant la confection de ces pâtes, laisse entendre qu'un tel usage remonte au moins aux Tang, il semble difficile de les considérer comme une création de la cuisine de montagne. À moins que l'on puisse retracer l'histoire de ce style culinaire jusqu'à cette époque. Du Fu, insistant d'ailleurs sur la « teinte adorable » de ce mets, estime qu'il est indispensable au prince par temps de canicule. Nous sommes bien loin de la simplicité rustique. C'est pourquoi Lin Hong ajoute que les Pâtes vertes sont tout aussi précieuses aux gens de la montagne. Il est ici moins question de goût que d'une harmonie entre la fraîcheur verte d'un plat d'été et l'environnement où il est confectionné ; et l'on retrouverait dans cette évocation une allusion implicite à la 
correspondance entre microcosme et macrocosme, si chère à la tradition chinoise. Une analyse sịmilaire pourrait être faite d'autres recettes, où l'entente avec la montagne, la nature et le cosmos comptent tout autant que l'ingrédient naturel et la simplicité des techniques de préparation.

Lin Hong est bien conscient que les circonstances peuvent en effet peser de tout leur poids sur l'appréciation des goûts et que la recherche de la saveur véritable suppose quelques conditions. Laissant la parole au ministre Su Yijian, il le rappelle dans la fable Délices d'une jarre glacée (Recette 6). Celui-ci, interrogé par l'empereur sur ses préférences alimentaires, commet une réponse surprenante : «Les aliments n'ont pas de goûts déterminés, ils sont bons pour qui en aime le goût. Pour moi, il n'y a rien de meilleur que le jus résiduel de la choucroute. » Dans la veine de Mengzi pour qui « tout est bon à celui qui a faim ${ }^{67}$, l'histoire raconte comment Su, réveillé en pleine nuit le gosier en feu après un excès de boisson, se précipite sur la première jarre venue et étanche ainsi une soif irrépressible. La leçon de $\mathrm{Su}$ Yijian est difficile à comprendre dans le contexte du $S J Q G$, car elle semble contrevenir aux règles énoncées précédemment. Se rassasier ainsi d'un sousproduit alimentaire, sans même prendre le temps de se laver les mains, comme le précise Su, ne s'accorde pas avec le souci de raffinement dont témoigne chaque recette de ce texte. Lin Hong a-t-il voulu rappeler que 1'homme est soumis à des contraintes physiologiques élémentaires, quel que soit son statut social, ou bien n'a-t-il pu résister à l'idée de raconter une belle histoire ? Quoi qu'il en soit, la jouissance simple et spontanée de Su Yijian nous conduit à réévaluer les normes du plaisir. Nul besoin parfois de grande dépense et d'ostentation pour jouir de l'existence.

67 Mengzi, Jinxin: « La nourriture paraît toujours agréable à ceux qui ont faim, et la boisson à ceux qui ont soif. Ils ne peuvent en bien juger; la faim ou la soif leur a gâté le goût » (Séraphin Couvreur [trad.], Les Quatre Livres IV. CEuvres de Meng Tzeu, Paris, Cathasia/Les Belles Lettres, s.d., p. 621). 


\section{L'esthétique quotidienne du montagnard}

\section{Un hédonisme de la tempérance}

Si la formule lévistraussienne «toute nourriture est bonne à penser » trouve une belle illustration, c'est bien chez Lin Hong. Le régime alimentaire que l'auteur du $S J Q G$ propose en une centaine de recettes règle non seulement les pratiques quotidiennes et festives des repas, mais définit aussi un certain mode de vie. Manger devient une métaphore du vivre. Mais il s'agit moins d' « être ce que l'on mange » que de « vivre comme on mange ».

Nous l'avons vu, la diète de Lin Hong s'adresse au reclus, à l'hôte des montagnes. Est-ce un lettré déchu, amer, exilé, contraint de se retirer de la société, qui ne lui aurait accordé aucune place à la mesure de ses talents ? Est-ce au contraire un poète, un joyeux luron qui, méprisant le jeu social, aspire à vivre libre parmi les siens, dans une harmonie sans prétention avec la nature? Les figures du reclus sont multiples, comme le montre A. Berkowitz, et Lin Hong ne se laisse pas facilement caractériser. Nous ne saurons jamais si c'est de sa propre volonté ou contraint par la nécessité qu'il se déclare «montagnard » et, à ce titre, révère son ancêtre $\mathrm{Lin} \mathrm{Bu}$, reclus célèbre. Montagnard ou pas, il ne fait aucun doute que l'auteur du $S J Q G$ et ses amis appartiennent à l'élite des junzi (hommes de bien), qui ne peuvent, en aucun cas, être confondus avec les xiaoren (hommes de peu). C'est pourquoi Lin Hong récrimine contre les cuisiniers vulgaires, qui croient bien faire en appariant les pousses de bambou à de la viande pour leur donner du goût, menaçant ainsi de pervertir l'homme de bien (huai junzi) avec des habitudes propres aux hommes de peu ${ }^{68}$. L'homme de peu fabrique le goût par un mélange vulgaire, tandis que l'homme de bien explore la douce amertume de la pousse de bambou dans une appréciation

68 Recette 21. Ce n'est pas l'association légume/viande qui est ici condamnée dans l'absolu, dans d'autres recettes elle est même conseillée (cf. Recette 34), mais celle toute spécifique bambou/viande; pour la raison probablement que la saveur du bambou est si fine qu'elle ne supporte pas d'être mariée avec celle plus forte d'un produit carné. 
sans partage. On voit là toute la différence entre la superficialité d'une saveur fabriquée, résultat immédiat de la technique, et la saveur authentique qui ne se donne pas d'emblée, mais qu'il faut aller découvrir dans la savouration.

Par cet exercice, Lin Hong se distingue de l'homme de peu, mais aussi de l'homme de bien ordinaire, le lettré en poste, le notable, voire le courtisan, dont le menu regorge des mets les plus sophistiqués. Les Galettes aux bourgeons de sapin (Recette 27), accompagnées d'un bon vin et dégustées en écoutant deux jeunes garçons chanter Le retour de Tao Yuanming, suffisent à déclasser les fleurons de la gastronomie impériale que sont les paumes d'ours et les bosses de chameau ${ }^{69}$. Tous les emblèmes de la montagne et du reclus sont évoqués : le maître de céans qui, malgré ses fonctions officielles ${ }^{70}$, a su adapter son maintien à la circonstance en adoptant le style de coiffure requis ; le vin, écho de la jarre qui attend Tao Yuanming; et surtout son plus célèbre $f u$, chant de ralliement des lettrés décidés à accomplir leur « grand vœu de retraite ${ }^{71}$. Dans cet ensemble de résonances, les Galettes sont nourritures d'autant plus symboliques qu'elles renvoient au pin, mentionné par deux fois dans Le retour, et lui-même symbole de la constance du solitaire qui trouve réconfort à vivre dans son voisinage.

Les signes extérieurs de l'adhésion à l'éthique montagnarde sont ici fort convenables et dans un sens à la portée du premier venu, beaucoup plus que ne le seront jamais les paumes d'ours et les bosses de chameau décriées par Lin Hong. De fait, les aspirations de Lin Hong sont bien calibrées. Dès le début de son ouvrage, Lin Hong pose ses marques. Il rejette la diète taoïste dans la recette de Riz sombre (Recette 1), dont il

69 Cf. Zhuang Shen, « Cong "bazhen" de yanbian kan Zhongguo yinshi wenhua yanbian », Bulletin of the Institute of History and Philology [Academia Sinica], 61.2, juin 1990, p. 433-479.

70 Il est enquêteur judiciaire à la Cour suprême (d'après Charles $O$. Hucker, A Dictionary of Official Titles in Imperial China, repr. Taipei, Southern Materials Center, 1988).

71 Pour la traduction et l'interprétation de ce poème, voir le travail de J.R. Hightower, op. cit. 
propose une version ordinaire pour l' "« accueil des invités à la montagne » et une autre spécialement destinée aux taoïstes adeptes de l' " abstinence des céréales $(b i g u) »$, régime trop radical qu'il ne saurait recommander.

La suite du texte confirme cette voie qui est aussi celle des enseignements du Huangdi neijing (Classique interne de l'Empereur Jaune) sur la nécessité d'absorber des céréales, et Lin Hong ne se laisse jamais entraîner par l'extrémisme taoïste, même s'il est parfois séduit par un rejet de la société. Choisissant la montagne, Lin Hong n'est pas pour autant un ascète, il entend simplement vivre en conformité avec les préceptes moraux de Confucius, ce que révèlent ses principes alimentaires. S'il ne cite jamais le maître, il mentionne Zhu Xi avec sympathie, et rappelle par la bouche de Ning Yuan, un lettré retiré, l'un des fameux enseignements du Lunyu : «Ne pas manger de viande plus que de céréale, voilà ce qui est véritable. ${ }^{72} \mathrm{Il}$ ne cessera de poursuivre la comparaison tout au long de son texte entre ceux qui ne respectent pas la parole du maître et les montagnards qui, au contraire, savent la mettre en pratique. Il stigmatise ainsi l'égoïsme des hommes de son temps qui n'ont plus la piété filiale d'un Gu Ao, resté célèbre pour ses cueillettes de riz sauvage que sa mère aimait tant (Recette 22). Il loue Cai Zun, le préfet de Wuxing, vertueux au point de planter amarantes ${ }^{73}$ et aubergines devant son studio pour assurer sa subsistance quotidienne et éviter ainsi de peser sur le peuple. Et Lin Hong de moraliser à ce propos : «Ceux de ses contemporains qui vaquaient à leurs affaires avec nonchalance, s'enivrant de bons crus et se repaissant de mets fins, comment pouvaient-ils ne pas avoir honte devant cette conduite exemplaire ?» (Recette 5).

Malgré les consignes de modération qui courent tout au long du $S J Q G$, le régime de Lin Hong n'a rien d'une pénitence, au sens chrétien du terme. Il est moins fondé sur le renoncement que sur la valorisation (ou la revalorisation, comme nous le verrons ci-dessous) de mets ou d'aliments, habituellement déconsidérés car trop vulgaires, trop sauvages, dans la mesure où ni la simplicité ni la rusticité n'ont jamais été des valeurs positives

72 Recette 40. Cf. Lunyu, «Xiangdang » : rou sui duo, bu shi sheng shi qi, et A. Cheng, op. cit., p. 83.

73 Amaranthus viridis L. 
pour ceux qui créaient les modes. Il n'est d'ailleurs nul besoin de faire de grands sacrifices pour suivre Lin Hong et l'on peut même en tirer quelque bénéfice sinon pour le portefeuille, du moins pour la santé.

Lin Hong feint l'étonnement lorsqu'il rencontre quelque notable gagné à sa cause qui sait fort bien faire la différence entre la saveur véritable des plats de montagne et les mets traficotés, les « nourritures de jade » (Recette 55) du beau monde. Ainsi de Huo Ru'an, que Lin complimente pour lui avoir offert un délicieux porridge aux pousses de bambou : «Cette façon de cuire respecte le goût des choses » (Recette 24) lui dit-il. De Zhang Yuezhai, homme d'État et homme de lettres, il fait un véritable éloge. Zhang non seulement se plaît dans la compagnie des « lettrés de la montagne, des forêts, des lacs et des mers " (shanlin huhai zhi shi), mais, en demandant au maitre de luth d' " accommoder correctement les fils d'argent ", il montre à tous qu'il connaît les principes de la cuisine de montagne (Recette 31). Par ailleurs, Lin Hong rappelle à ceux qui n'auraient pas compris sa leçon que les nourritures de montagne sont autrement plus efficaces que les drogues dispendieuses lancées par la mode : mieux vaut manger de la courge calebasse à la vapeur avec un peu de sauce jiang que de compter sur une quelconque technique alchimique pour se sentir en forme (Recette 7); et à ce propos, les Gâteaux de riz à l'armoise blanche $e^{74}$ sont tout autant, sinon plus fortifiants que corne de cerf et stalactite, pourtant si cotées.

S'il est difficile de mesurer le succès de la gastronomie de Lin Hong hors de son milieu, puisque nous n'avons guère les moyens de vérifier ses dires, elle ne semble pas toujours aussi hétérodoxe qu'il veut bien l'affirmer. Il se flatte d'ailleurs que certaines recettes de son répertoire soient appréciées jusqu'à la Cour, comme si tout ce qui touchait de près ou de loin à la personne physique de l'empereur acquérait naturellement un prestige, même aux yeux d'un adepte convaincu comme l'auteur du $S J Q G$. Comment ne pas croire

74 Recette 59. Je n'ai pu identifier la plante baipeng, utilisée dans cette recette, et que j'appelle « armoise » par commodité. Dans les dictionnaires consultés, on trouve l'entrée penghao qui est soit Chrysanthemum coronarium L. var. spatiosum Bailey, soit Artemisia sieversiana Ehrh ex. Willd, cette dernière plante étant aussi appelé baihao. Ma traduction provisoire est donc fondée sur l'hypothèse d'une neutralisation des deux appellations penghao et baihao, mais rien n'est moins sûr. 
doublement à l'efficacité de la Liqueur de rosée (Recette 41) que Zhang Yizhai offrit un soir à ses invités et qui dissipa leur ébriété, sans même qu'ils s'en rendent compte, quand la recette de ce mystérieux breuvage provient du palais impérial ? On ne sait si la Cour s'est inspirée parfois des pratiques montagnardes ou, à l'inverse, si ce sont les montagnards qui ont repris quelques mets de la Cour pour les arranger à leur manière, quoi qu'il en soit, le Chausson mer-montagne (Recette 37), "souvent servi à la Cour » sous une autre appellation, n'en gagne que plus d'éclat. L'empereur d'ailleurs, pourtant à des lieues par sa fonction de l'idéal promu par Lin Hong, peut même jouer le rôle de modèle quand il adopte un plat rustique et entend ainsi encourager l'abstinence de chair : " Dans ce cas, comment nous les montagnards pourrions-nous vivre dans le luxe ?»(Recette 35), constate notre auteur. La voie gastronomique et culinaire proposée dans le $S J Q G$ est étroite, elle s'écarte des abstinences radicales du taoïsme, comme elle se passe du luxe inutile de la Cour et des puissants. C'est un idéal gourmand tout de mesure et de tempérance.

\section{La tentation végétarienne}

Le $S J Q G$ n'est pas un traité de cuisine végétarienne, car il contient quelques recettes de viandes et poissons. Cependant, on ne peut manquer d'être frappé par l'inclination de son auteur à faire l'éloge de la consommation de végétaux, et, inspiré probablement par Su Dongpo ou d'autres poètes des Song, à s'intéresser tout particulièrement à des légumes très communs. Lin Hong rapporte avec vénération un vers de Su vantant l'ineffable goût d'un potage à la petite rave : « Ce ne peut être qu'un nectar des dieux, car un tel délice ne peut exister sur terre ! (Recette 16). Certes, on perçoit chez le grand poète un souci d'anticonformisme qui le pousse à vanter justement ce que tout le monde (c'est-à-dire ses pairs, les lettrés bon teint) méprise ; un jour il porte aux nues les haricots, une autre fois il crée une recette à base de viande de porc qui restera célèbre dans les annales de la gastronomie chinoise (Dongpo rou $)^{75}$. Si Lin Hong lui emboîte le pas, il

75 Dans l'un de ses poèmes écrits durant son exil à Huangzhou, Su Shi raconte comment il achetait à vil prix de la viande de porc, car les riches ne voulaient 


\section{Françoise Sabban}

cherche néanmoins à justifier sa préférence pour ces mets trop pauvres, et surtout à convaincre ses lecteurs du bien-fondé de son choix. Car l'auteur du $S J Q G$ ne parvient pas vraiment à se détacher de la longue tradition qui attribue un rôle prestigieux ${ }^{76}$ à la viande en tant que nourriture des hommes de bien, tandis que le bas peuple est associé à la consommation de légumes. Il souligne que l'expression « teint de légume " (caise) $)^{77}$ désignait un crèvela-faim, appelé ainsi par métonymie, et il rappelle que si les Anciens louaient les légumes, c'était dans l'intention d'inciter les lettrés à en connaître le goût et à faire en sorte que le peuple n'en ait justement pas le teint (Recette 74). Bref, les légumes n'avaient pas vraiment bonne presse et Lin Hong montre bien qu'il suffit de servir à quelqu'un un plat de légumes indigne de son rang pour le déconsidêrer ou l'humilier. L'histoire de Xue Lingzhi rapportée ici est exemplaire. Précepteur à la cour des Tang, et ne supportant plus la maigre pitance de germes de luzerne ${ }^{78}$ qu'on lui allouait quotidiennement, Xue exprima son mécontentement en un poème allusif qu'il écrivit imprudemment à la vue de tous. La plainte n'échappa pas à l'empereur, qui lui répondit aussitôt par la même voie qu'il était libre de quitter le palais s'il ne trouvait aucune satisfaction à vivre dans ses murs. Ce que fit Xue, effrayé par la colère impériale

Certes, les légumes et les plantes ont des qualités diététiques que Lin Hong ne manque pas de mettre en avant, mais il sait bien que la saveur et la jouissance du palais inspirent plus souvent les choix alimentaires des hommes que la recherche de la santé ; aussi s'efforce-t-il toujours de louer le goût de tel ou tel légume. Ayant à peine appris de Zheng Weibin le secret

pas en manger et les pauvres ne savaient pas la préparer, pour confectionner un ragoût dont il avait le secret. Cf. à ce propos, Zhou Zizhi (1131-1162), Zhupo shihua (Propos sur la poésie du Sieur Colline aux bambous), cité dans Lin Zhengqiu et al., Zhongguo Songdai caidian gaishu, p. 36-38.

76 Cf. F. Sabban, « La viande en Chine », p. 79-90.

77 Nous dirions en français « teint de navet » pour la même image.

78 Recette 3. Muxu, Medicago sativa L. : l'image est forte en français, cette plante étant exclusivement considérée comme un fourrage. C'est pourquoi on ne relève jamais cette dénomination dans les circuits commerciaux alimentaires français, où les pousses sont parfois vendues sous l'appellation anglaise " alfalfa ». 
d'une poudre d'épices «spéciale cuisson des légumes », il l'essaie et, la trouvant si parfumée, il évoque par association d'idées le général Tang Xiaoxin, amateur de légumes au point de les préférer aux meilleurs morceaux des meilleures viandes. Comment un militaire, par métier voué à sacrifier des vies humaines, n'emporterait-il pas l'adhésion lorsqu'il prétend épargner la vie animale pour se nourrir (Recette 74) ? On voit là tout l'art rhétorique de Lin Hong, qui, pour convaincre, va même jusqu'à s'effacer derrière la carrure d'un général.

Cependant cette valorisation du légume repose en réalité sur un paradoxe, car le légume ne vaut pas toujours pour lui-même dans le $S J Q G$. On se souvient par exemple que les haricots de Su Dongpo sont « fondants comme du beurre ", l'un des sous-produits animaux les plus côtés à l'époque ${ }^{79}$. Et l'intérêt culinaire que présente un légume aux yeux de Lin Hong apparaît bien souvent dans la comparaison de son goût avec celui de la viande, celle-ci restant la référence majeure en termes de gastronomie.

Curieusement, la cuisine végétarienne chinoise repose sur cette ambiguité dont elle a su merveilleusement tirer parti. La place prééminente accordée à la chair animale, appréciée pour ses qualités gustatives, a favorisé le développement d'une recherche de succédanés de la viande à base végétale, puisque pour diverses raisons d'ordre religieux ou éthique le besoin s'est fait sentir d'une cuisine qui puisse conjoindre gastronomie et respect de certains préceptes et interdictions alimentaires. Le $S J Q G$ rend compte à sa mesure de cette évolution dans six de ses recettes, que l'on peut qualifier de « végétariennes », soit que leurs composants relèvent du répertoire classique de la cuisine végétarienne, soit que leur dénomination l'indique explicitement : ainsi de la recette du Canard végétarien, une courgecongourde à long col cuite à la vapeur (Recette 19), ou de la Gelée de poisson végétarienne pour accompagner le vin, une gélatine d'agar-agar taillée en fines lanières censées imiter le sashimi de poisson (Recette 99),

79 Cf. Wang Saishi, « Songren shi su qutan » (À propos de la consommation de beurre sous les Song), Zhongguo pengren, 6, 1995, p. 14-15 ; voir aussi F. Sabban, «Un savoir-faire oublié : le travail du lait en Chine ancienne », Zinbun. Memoirs of the Research Institute for Humanistic Studies, Kyoto University, 21, 1986, p. 31-66. 
ou encore de la Viande frite factice, une julienne de gluten et de chair de courge frite à la poêle dans du saindoux avec des épices (Recette 64). Dans ce dernier exemple, le mot jia « factice » est un terme technique culinaire, un quasi-synonyme de $s u$ « végétarien, maigre »; on le relève fréquemment dans certains textes Song mentionnant des noms de plats ${ }^{80}$, où il suggère très certainement la présence d'un ersatz de viande, du type du gluten. Fait remarquable, cette dernière recette tolère l'usage d'une graisse animale, ce qui laisse supposer que le champ sémantique du terme $s u$ n'est pas clairement défini.

L'identification des trois autres recettes est moins immédiate, mais elle devient évidente dès qu'on en lit le texte. Le Mou de jade farci (Recette 35) relève ici de la cuisine d'imitation, puisqu'il ne s'agit pas d'un plat de triperie comme le suggère son nom : le mou (poumon) est modelé dans une pâte faite de fécule, de beignets écrasés, de graines de sésame, de pignons, de noix et de sucre, parfumée à l'aneth ${ }^{81}$ et colorée en rouge avec du ferment hongqu. Il est ensuite cuit à la vapeur, puis découpé en «imitant des morceaux de mou ». On retrouve le $\mathrm{Mou}$ farci, dans sa version authentique comme dans son interprétation maigre et factice, dans les traités culinaires Yuan, où il semble être un plat valorisé ${ }^{82}$. La recette de Raviolis surpassant la viande ne fait guère mystère ; les raviolis sont garnis d'un hachis de pousses de bambous, de champignons et de fruits secs, considéré comme succulent, et qui leur vaut cette désignation (Recette 85). La Bardane séchée, quant à elle, est une racine aromatisée et traitée par dessiccation, qui a le " même goût que la viande séchée " (Recette 96).

De ces six recettes, il n'y a que le Mou de jade farci qui suscite de la part de Lin Hong un commentaire d'ordre moral, évoquant l'interdiction bouddhique d'atteinte à la vie. Ayant remarqué que ce plat était aussi confectionné dans les cuisines impériales et qu'il recevait alors la flatteuse désignation de « Mou de jade farci aimé de l'empereur », il conclut : « $\mathrm{Ce}$

80 Cf. par exemple les textes décrivant les capitales des Song du Nord et des Song du Sud. Cf. ci-dessus, note 6.

81 Sesamum indicum D.C., Pinus koraiensis Sieb. et Zucc., Juglans regia L. ? ou Juglans cathayensis Dode ?, Anethum graveolens L.

82 On peut émettre l'hypothèse que l'existence d'une version grasse et maigre d'un « même » plat signifie qu'il était très apprécié. 
n'est tout compte fait qu'un plat végétarien. Mais il prouve que l'empereur est sobre et a le désir de respecter la vie. Dans ce cas, comment nous les montagnards pourrions-nous vivre dans le luxe ? "

Il est difficile d'évaluer la place de ces recettes dans l'économie générale du $S J Q G$, et, à la limite, il paraît artificiel de les distinguer d'un ensemble qui est déjà très inspiré par le végétal. Pourtant, elles révèlent la curiosité de Lin Hong pour ce qui semble être, déjà à son époque, un véritable style culinaire apprécié pour la variété de ses saveurs et la complexité de ses techniques vouées à l'imitation des chairs animales et de leur goût.

Car Lin Hong est partagé, sinon déchiré. Tout de même, il n'est pas encore prêt à renoncer aux délicieux crabes qui accompagnent si bien le vin, ou à se passer d'une dégustation surprise de lièvre en fondue (Recette 38). S'il cite Lu You, mangeur d'igname sur la fin de sa vie et quasiment en jeûne permanent (jin wei chang zhai) (Recette 66), ou encore s'il rappelle que son ancêtre Lin Hejing trouvait quelque peu cruel de manger les canards et les faisans, dont le plumage est si joli (Recette 68), il ne désavoue pas les enseignements du Bencao quand la leçon lui convient; la chair du cerf hydropote est supérieure à celle de mouton, dit le texte canonique. Et comme pour conforter le Bencao, Lin Hong ajoute que les taoïstes la soumettaient à dessiccation (Recette 90); remarque qui est loin d'être innocente, si l'on songe que la viande séchée est l'un des aliments de prédilection de certaines diètes taoïstes restrictives, et dans ce sens une nourriture précieuse et indispensable pour qui veut modérer sa consommation alimentaire. C'est aussi une chair de gibier noble qui n'a rien de commun avec les vulgaires produits d'un élevage ordinaire. Ainsi, le porc ne semble pas être du goût de Lin Hong. Il ne le mentionne qu'une fois à propos de la fondue de lièvre en précisant que ce type de plat peut se préparer également avec du porc ou du mouton. Cette désaffection pour le porc n'était pas propre à Lin Hong, nous l'avons déjà évoquée à propos de Su Dongpo, qui va à l'encontre des préjugés de son époque en mettant au point sa célèbre recette de ragoût. La viande de porc semblait en effet méprisée par les hommes de bien, tandis que la viande de mouton était au contraire très appréciée, si l'on en croit les descriptions du Dongjing menghua lu (Rêve de la splendeur de la capitale orientale déchue) et du Mengliang lu qui mentionnent de nombreux plats à 
base de mouton ${ }^{83}$. Lin Hong, contrairement à Su Shi, est en accord avec l'esprit de son temps. Ainsi, le cochon ne fait pas partie de la liste des animaux recommandés pour un élevage à la montagne mentionnée dans le Shanjia qingshi ${ }^{84}$. Par ailleurs, dans le même texte, il explique qu'il s'est interdit de consommer du porc en raison d'ennuis de santé, et que depuis il s'en trouve beaucoup mieux ${ }^{85}$.

\section{La diététique de Lin Hong}

Hormis cette confidence réservée aux lecteurs du Shanjia qingshi, Lin Hong n'évoque jamais ses petits soucis de santé personnels dans le $S J Q G$; mais il n'est nullement indifférent à l'action thérapeutique de certains aliments, puisqu'une trentaine de ses recettes contiennent une remarque ou un conseil d'ordre diététique.Toutefois, à aucun moment Lin Hong n'exprime la moindre conception générale qui nous permettrait de comprendre quelles sont ses idées en la matière. Ses considérations diététiques sont conjoncturelles et ne forment pas système. Ce sont des indications précises pour telle ou telle affection : des Huntun à la racine de cédrèle (Recette 15$)^{86}$ pour soigner la diarrhée (li) et les lombalgies (yaotong); du Chat sauvage (Recette 93$)^{87}$ pour apaiser les hémorroïdes (zhi); des Pâtes botuo à la rehmannia (Recette 13) ${ }^{88}$ pour soulager les nausées (xintong) et éliminer les « amas de vers » (chongji); de la Ciboule de Chine à feuilles lancéolées (Recette 26$)^{89}$ pour guérir la strangurie et l'anurie ( $z h$ lin $b i$ ). Ou bien elles se présentent comme un simple rappel des facultés thérapeutiques de certains produits, ainsi du pouvoir réchauffant et tonifiant (wenbu) du taro (Recette 25$)^{90}$, de l'action désopilante (tong

83 Cf. note 6.

84 Cf. Shanjia qingshi, Zhong mei yang he tuji, 22/31b.

85 Cf. Shanjia qingshi, Shi tun zi jie (Interdiction personnelle de manger du porc), 22/31a.

86 Toona sinensis (A. Juss.).

87 Felis bengalensis Kerr.

88 Rehmannia glutinosa (Gaertn.).

89 Allium tuberosum Rottler.

90 Colocosia esculenta (L.) Schott. 
xinqi, « débloquer le souffle du cœur ») des petites raves (Recette 62) ${ }^{91}$, du caractère apaisant (anxin) de la bourse-à-pasteur (Recette 61) ${ }^{92}$. Mais Lin Hong reste cuisinier jusque dans ses recettes médicinales, qui relèvent d'une véritable cuisine, même si celle-ci a des vertus curatives ; l'aliment cuisiné y est considéré d'abord comme un nutriment, un nutriment un peu particulier qui possède une valeur thérapeutique par lui-même ou qui l'acquiert grâce à un autre ingrédient qu'on lui ajoute. Il faut d'ailleurs souligner que ces recettes mentionnent rarement une posologie, comme c'est souvent le cas dans les bencao ${ }^{93}$ (Materia medica), ce qui ne distingue pas ce sousensemble de recettes médicinales du traité, dont l'orientation générale est plus culinaire que diététique.

Lin Hong connaît aussi certaines incompatibilités entre aliments spécifiques qu'il ne faut en aucun cas manger ensemble, sous peine de tomber malade ou de nuire à telle ou telle fonction organique. Ces interdits alimentaires font l'objet de longues listes dans les calendriers et dans les ouvrages diététiques postérieurs ${ }^{94}$. Les interdits cités par Lin Hong sont aussi répertoriés dans le grand codex contemporain Zhenglei bencao (Materia medica encyclopédique ${ }^{95}$ : ainsi, le crabe et le kaki ${ }^{96}$, consommés au même

91 Raphanus sativus L.

92 Capsella bursa-pastoris (L.) Medic.

93 Ainsi, le verbe $f u$ « prendre, avaler », de même que certaines spécifications chiffrées indiquant le nombre de prises conseillées, apparaissent rarement, même dans ces contextes. On pourrait d'ailleurs se demander si l'emploi de $f u$ ne signalerait justement pas l'orientation fortement médicinale d'une recette. $\hat{A}$ ce propos, comparer la Recette 1 , où l'on emploie le mot $f u$, avec la recette 52 , où c'est le verbe $s h i$ « manger » qui apparaît pour une indication similaire : jiu fu yan nian yi yan, "si l'on en prend pendant longtemps, cela prolonge la vie et profite au teint ", et jiu shi keyi ming mu yan nian, «si l'on en mange pendant longtemps, cela peut éclaircir la vue et prolonger la vie ».

94 Cf. par exemple, le Yinshan zhengyao (Les principes corrects du boire et du manger), 1330, de Hu Sihui, juan 2, Shiwu xiangfan (Les aliments qui se correspondent et qui s'opposent), Li Chunfang (éd.), Yinshan zhengyao, Pékin, Zhongguo shangye chubanshe, 1988, p. 204-206.

95 Chongxiu Zhenghe jingshi zhenglei beiyong bencao (Materia medica encyclopédique et pratique basée sur les ouvrages classiques, nouvellement révisée, de l'époque Zhenghe), (éd. 1249), Tang Shenwei comp., reprint Pékin, Renmin weisheng chubanshe, 1957. Désormais ZLBC.

96 Shi, Diospyros kaki L. 


\section{Françoise Sabban}

repas, contreviennent à ceux qui souffrent des « vers du vent ${ }^{97}$ (fengchong) selon Lin Hong, tandis que pour les auteurs du $Z L B C$ ces deux aliments mangés ensemble donnent « des douleurs de ventre et une grande diarrhée » (fu tong da xie $)^{98}$; de même, le faisan ${ }^{99}$ associé à des noix et à des oreilles de Judas ${ }^{100}$ provoque une « descente de sang » pour Lin Hong, ce que le $\mathrm{Z} L B C$ attribue précisément à une « crise des cinq hémorroïdes » ( $\mathrm{fa} w u z h i$ li xia $x u e)^{101}$; quant aux raisons de l'incompatibilité du lapin et du poulet (Recette 38), Lin Hong n'en dit rien, tandis que les auteurs du $Z L B C$ pensent que l'absorption simultanée de ces deux viandes donne un teint jaune (mian fa huang) $)^{102}$.

À l'exception de la Confiture sèche d'ophiopogon (Recette 63), qui est manifestement médicinale - elle irrigue et tonifie (ziyi) - et de quelques autres recettes comme celle des Pâtes botuo à la rehmannia (Recette 13), Lin Hong ne formule pas ses recettes en fonction d'une indication thérapeutique donnée d'emblée. Celle-ci est généralement signalée au cours de l'énoncé du texte, comme une information utile supplémentaire. Cependant, l'auteur du $S J Q G$ se sert parfois avec habileté de ces renseignements pour surenchérir sur des qualités proprement gastronomiques, ainsi des Feuilles de jade (Recette 28), rôties bien croustillantes, qui plus est, contribuent à stopper les glaires (zhi tan) tout en améliorant la digestion (huashi). Deux mets seulement, le Riz sombre (Recette 1) et le Riz doré (Recette 52) sont réputés «prolonger la vie», et encore ce ne sont pas leurs seules indications. Lin Hong propose deux modes de préparation de Riz sombre ${ }^{103}$ : le premier consiste à confectionner une espèce de riz de

97 Recette 49. L'éditeur du texte (cf. ci-dessus note 9) ne comprenant pas ce que Lin Hong entend par fengchong propose une autre traduction : "Les crabes étant porteurs de parasites, il ne convient pas de les manger avec des kakis. "

$98 Z L B C$, chap. 23, p. 426, xia.

99 Zhi, Phasianus colchicus torquatus Gmelin. Cf. Recette 68.

100 Muer, Auricularia auricula (L. ex Hook.) Underw.

$101 Z L B C$, chap. 19, p. 403, shang.

$102 Z$ ZLBC, chap. 17, p. 385, shang.

103 Cette préparation a une longue histoire. Elle est mentionnée dans le $Z L B C$ (chap. 14,p. 350, shang) à l'entrée de la plante nanzhu, sous le nom de wufan, et les propriétés de la plante elle-même sont celles qui sont attribuées par Lin 
conserve, en faisant d'abord macérer la céréale dans le jus de la plante Vaccinium bracteatum Thunb. (nanzhu mu) pour en teinter les grains, puis à leur faire subir une précuisson en vue de leur utilisation future selon les besoins. Le second, qualifié de xianfang (recette d'immortalité), s'adresse, comme le précise Lin Hong, à ceux qui « à l'instar de Zifang désirent s'abstenir de céréales (bigu) » ${ }^{104}$. Allusion est faite ici à Zifang, ou Zhang Liang (?-185 avant J.-C.), compagnon d'armes de Liu Bang, et qui, malade, choisit de suivre la voie taoïste en pratiquant la diète de l'abstinence des céréales $^{105}$. Il s'agit cette fois-ci de faire tremper du millet (Setaria italica (L.), qing jing mi) dans une variété d'argile siliceuse bleue (qing shizhi) pendant trois jours, puis de le façonner en petites boulettes de la taille d'une prune. Cette curieuse préparation montre que l'abstinence de céréale est toute relative, même si, à en croire la posologie, très précise dans ce cas, l'absorption est limitée à une ou deux boulettes ( fu yi er wan ke bu ji), c'est tout de même une céréale que l'on mange. La préférence de Lin Hong pour la première préparation, qui lui semble adaptée à ses propres choix de vie, suggère qu'il ne s'intéresse guère à l'immortalité et à la longévité, façon taoïste, et qu'il reste plus préoccupé d'élaborer une diète parfaite, tenant l'équilibre entre le bon pour la santé et le bon pour les sens - deux exigences qui ne lui semblent nullement inconciliables, dès lors que l'on s'astreint à jouir du « goût véritable » des aliments et que l'on mange avec modération.

Par ailleurs, si l'on examine les vertus thérapeutiques qui sont attribuées à la vingtaine de plantes citées en les comparant aux codex habituels, on

Hong à la préparation: jiu fu qing shen chang nian ling ren bu ji « absorbée pendant longtemps, elle allège le corps, prolonge la vie et supprime la sensation de faim ». Selon Wu Ke, l'éditeur actuel du SJQG (p. 1, note 1), ce sont les taoïstes qui ont contribué à la réputation de longévité du Riz sombre. Toujours d'après Wu Ke, à l'époque des Qing le wufan fut considéré comme un mets festif à Hangzhou, où on le préparait sous forme de gâteaux pour l'entrée de l'été.

104 À propos de la diète taoïste, cf. Jean Levi, « L'abstinence des céréales chez les taoïstes ", Études chinoises, 1, 1983, p. 3-47.

105 Voir la biographie de Zhang Liang dans le Shiji, chap. 55, « Liuhou shijia », 25, éd. Zhonghua shuju, 1975, p. 2033-2049. 


\section{Françoise Sabban}

voit que Lin Hong s'est inspiré de ses prédécesseurs, mais qu'il a su parfois innover. Ainsi par exemple dans la recette Ciboule de Chine à feuilles lancéolées (Recette 26), la ciboule est considérée comme un agent thérapeutique dans les maladies urinaires, ce que personne avant Lin Hong $n^{\prime}$ 'avait signalé ${ }^{106}$. Lin Hong est un homme libre et, s'il poursuit une tradition dont il reprend les acquis tout en lui ajoutant à l'occasion les fruits de son expérience, il cite à son gré les bencao sans en préciser toujours l'origine, et il lui arrive aussi de montrer son esprit critique ou son humour à l'égard de certaines indications, quand les faits ne se révèlent pas à la hauteur de l'efficacité promise : reprenant une prescription ordinaire sur l'interdiction de manger de la petite rave avec de la rehmannia sous peine de voir blanchir prématurément ses cheveux, il se demande si son ami Jingyi, grand amateur de petites raves et dont la tête est déjà chenue malgré son jeune âge, a vraiment enfreint l'interdiction (Recette 62)! Posant ainsi la question, il insinue le doute quant à la véracité de l'affirmation du codex canonique. Le $S J Q G$ témoigne justement de la somme de connaissances qu'un homme de lettres, plus poète que naturaliste, possédait à son époque, et on peut probablement le considérer à ce titre comme une vulgate diététique partagée par tout homme cultivé, préoccupé de son hygiène et de son mode de vie.

La nature bâtarde du $\mathrm{S} J Q G$ en fait une source utile pour qui s'intéresse à la vie quotidienne du lettré retiré et à ses motivations. Bien que ce texte ne contienne aucune description systématique des activités à la montagne, et que son auteur n'expose jamais de façon didactique ses conceptions en matière d'hygiène de vie et d'alimentation, une lecture attentive permet d'expliciter les principes qui guident Lin Hong et ses amis. Aussi curieux que cela puisse paraître, la retraite n'implique nullement l'isolement et la séparation complète du monde. Pas plus qu'elle n'exige de déchirants renoncements ou de dures privations alimentaires. Certes, le lettré retiré ne

106 Pour d'autres exemples, cf. Fang Chunyang et Xu Shumin, « Shanjia qinggong de shiliaoxue jiazhi » (Valeur sur le plan de la nutrition du traité Les vivres simples du montagnard), Zhonghua yishi zazhi, 13.3, 1983, p. 183-184. Je remercie Ute Engelhardt de m'avoir donné une photocopie de cet article. 
vit plus dans la société de ses pairs, mais il n'en est pas coupé. Installé dans sa «maison de montagne », il organise une société parallèle avec ses propres règles, dont les interactions sont multiples avec ceux qui continuent d'assumer une charge de l' « autre côté ». La commensalité semble être au cœur de cette vie érémitique d'un genre particulier, la pleine appréciation des mets et des boissons n'étant envisagée que dans l'échange, lui-même petite part d'interrelations beaucoup plus larges. Car repas et beuveries n'ont pas pour unique finalité l'assouvissement de la gourmandise. Si l'on déguste dans la jouissance modeste, si l'on s'enivre même, c'est toujours à l'occasion d'une rencontre, d'une promenade, d'une visite, d'un voyage... Toute activité collective est conçue comme une totalité d'expériences sensuelles simultanées dans une communion avec la nature : l'idéal étant d'être assis par une belle journée sous de fraîches frondaisons mêlées de senteurs florales, le regard comblé par quelque tableau accordé à la saison, de composer de la poésie, d'écouter de la musique ou de plaisanter, tout en étant régalés de mets délicats ou de généreuses rasades du meilleur cru. Tout est pondéré, tout est mesuré, et hormis l'ébriété permise que l'on dissipera par quelque breuvage médicinal, la gourmandise reste bridée et ne conduit pas à la goinfrerie. Cette vie de dandy à l'hédonisme maîtrisé nécessite une réelle infrastructure matérielle dont Lin Hong donne quelque idée dans son Shanjia qingshi, où il énumère le personnel qu'il convient d'engager pour l'installation d'une maison de montagne : un cuisinier, un serviteur, une servante, deux jardiniers ${ }^{107}$. Au détour d'une recette, Lin Hong mentionne rapidement la présence de sa femme, qui a mis la main à la pâte et qui reçoit à l'occasion un conseil pour améliorer le potage de chou qu'elle vient de préparer (Recette 74). Les plats les plus raffinés sont toujours confectionnés ou servis par les domestiques de l'hôte, prêts à répondre aux ordres de leur maître. Nous sommes loin du préfet Cai Zun, le modèle de vertu chanté par Lin Hong, qui cultivait de ses propres mains les quelques légumes nécessaires à sa subsistance, pour éviter de peser sur la collectivité ! Dans un essai bref mais féroce, Lu Xun épingle sans ménagement ces hommes aux aspirations prétendument élevées qui, dit-il, n'auraient pu survivre sans leurs esclaves pour bêcher la terre à leur place.

107 Zhong mei yang he tu ji, 22/31b. 
Pour Lu Xun, l'ermitage représentait tout simplement un autre choix de carrière et il conseille de ne pas se laisser abuser par les beaux discours de ces messieurs sur les plaisirs du travail rustique ${ }^{108}$.

Par ailleurs, si Lin Hong semble avoir beaucoup voyagé, parcourant comme ses amis sentiers et chemins de montagne, la question se pose de savoir en quel type de lieu il recommande d'élire domicile. Si l'on s'en tient à sa cuisine et à ses ingrédients, il ne fait guère de doute que la maison du lettré retiré devait être située à une distance raisonnable d'une ville industrieuse ${ }^{109}$, source d'approvisionnement des ingrédients indispensables à l'expression de son talent culinaire. Ainsi, ce n'est pas dans la nature lointaine et sauvage des pics, des rapides et des forêts qui effrayaient les hommes ${ }^{110}$ que Lin Hong s'est réfugié, mais dans un cadre naturel plus conforme à ses besoins. De fait, l'inventaire des produits comestibles cuisinés dans le $S J Q G$ suggère le type d'environnement choisi par Lin Hong et reflète en même temps le véritable statut de la diète qu'il préconise. Un grand nombre de plantes de cueillette, des légumes du jardin, des céréales, quelques gibiers, un peu de venaison, des poissons d'eau douce et tous les adjuvants de la grande cuisine constituent la base d'un régime alimentaire qui se distingue à la fois des pratiques taoïstes, trop ascétiques et solitaires, et des menus très carnés des riches et des puissants. Diète de juste milieu qui concilie le plaisir de la gastronomie, la tempérance, la diététique, elle ne peut être strictement végétarienne dans la mesure où elle opère en vérité

108 Lu Xun quanji, Qiejieting zawen er ji, yinshi, Pékin, Renmin wenxue chubanshe, 1973, vol. 6, p. 227-229. Cf. aussi Li Chi (op. cit.), qui montre comment avec les siècles le choix de la retraite a souvent fini par signifier l'opportunisme.

109 Pour une époque postérieure, c'est la « montagne » proche, celle des faubourgs parfois, ainsi que l'explique Li Chi (op. cit., p. 245) à propos du poète Yuan Mei (1716-1797), qui «entra dans les montagnes » (ru shan), selon sa propre expression, s'installant en fait dans le jardin Suiyuan, une magnifique propriété, sise dans la banlieue de Nankin, à deux pas de la vie trépidante de la ville.

$110 \mathrm{Cf}$. à ce sujet l'évolution des significations attachées à la montagne, dans Paul Demiéville, «La montagne dans l'art littéraire chinois », France-AsieAsia, 20, 183, 1965, p. 7-32. 
une transposition de la Nature jusque dans l'assiette. En valorisant l'authenticité des saveurs, en privilégiant le végétal, qu'il soit sauvage ou cultivé, enfin en distinguant le produit de la pêche, et surtout la viande de chasse dont la sauvagerie est habilement neutralisée par des modes de cuissons très étudiés, la diète de Lin Hong illustre ainsi parfaitement l'éthique modérée du montagnard, lui-même habitant d'un havre de paix façonné selon ses aspirations, jouissant du confort nécessaire à une vie sociale agréable, frottée de quelques senteurs rustiques, mais dégagée de toute responsabilité pesante.

En raillant les «faux ermites » dont la vertu ne serait qu'opportunisme bien placé, $\mathrm{Lu}$ Xun joue son rôle de moraliste, mais il révèle en même temps la véritable nature du $S J Q G$ qui sonne comme le manifeste d'une société du refus parfaitement intégrée dans les allées de la culture dominante et dont les valeurs sont le reflet inversé de celles habituellement adoptées par le plus grand nombre. De fait, ce texte n'a rien d'utopique, il témoigne d'un mode de vie choisi par un groupe rejetant l'opulence, la puissance, l'ostentation, signes reconnus de la réussite sociale et de la place que l'on occupe dans son temps, en Chine comme ailleurs. Pourtant Lin Hong n'est en aucune façon un marginal ostracisé par ses pairs, un misanthrope dont la hargne à l'égard de son prochain se nourrirait des griefs suscités par la fréquentation du monde ou encore un artiste incompris que le génie créateur pousserait à s'isoler. Que les règles de fonctionnement de la " société " à laquelle appartient Lin Hong exigent certains sacrifices, notamment alimentaires, ne transforme nullement ses membres en exclus du système. Leur façon de vivre apparaît au contraire comme un idéal auquel, au fond, tout homme de bien aspire secrètement, et Lin Hong cite plusieurs exemples de puissants qui, malgré leurs charges et leur responsabilités, se complaisent dans la compagnie des « montagnards ». L'esthétisation du quotidien dont témoigne le $S J Q G$ ne rompt d'ailleurs pas avec l'esthétique ordinaire du lettré, elle s'exprime dans un autre registre. À cet égard, le choix du renoncement librement consenti par Lin Hong, en même temps magnifié par un hédonisme tempéré, est en tout point opposé à l'abstinence périodique imposée par l'orthodoxie religieuse dans l'Europe chrétienne à la même époque. Les jeûnes auxquels contraignait l'année liturgique apparaissaient 


\section{Françoise Sabban}

comme de véritables pénitences et l'étaient d'autant plus qu'on occupait le bas de l'échelle sociale et qu'on ne disposait d'aucun moyen de les adoucir ${ }^{111}$. Pour ceux qui les acceptaient par dévotion ou conformisme, ils ne prenaient sens que dans la mortification, et ne pouvaient en aucune façon être l'objet d'un souci esthétique. Il n'existait pas de voie possible entre le débordement des appétits et la sobriété, entre la luxure et la chasteté. Et quand la ferveur religieuse venait à manquer, une stricte diététique, qui bannissait les excès et préconisait le jeûne à tout propos pour favoriser l'élimination des superfluités, prenait le relais, menant à une triste tempérance, voire à l'abstinence la plus totale ${ }^{112}$. Il n'est donc rien de plus dépaysant pour nous que la joyeuse misanthropie de Lin Hong, qui ignore l'acariâtre et transforme en plaisir ce qui nous paraît pénitence ou châtiment.

111 Cf. Carole Lambert, «La nourriture comme signe de distinction religieuse et sociale de Thomas d'Aquin à Érasme ", Heresis, 26-27, 1996, p. 99-113.

112 Cf. l'exemple du peintre florentin du Xvi $\mathrm{e}^{\mathrm{e}}$ siècle, Jacopo Pontormo, dont la diète des dernières années de sa vie est entièrement inspirée par le souci obsessionnel de son corps et la peur de la mort, qu'il résout par des jeûnes périodiques, censés rétablir les fonctions corporelles empêchées par les encombrements dus aux excès alimentaires et à l'abus de boisson, Jean-Claude Lebensztejn (trad., éd.), «Le journal de Pontormo », Dossier Pontormo, Macula, 1974, p. 4-38. 


\section{ANNEXE}

\section{Note sur la traduction des titres de recettes}

La nature du $S J Q G$ et sa compréhension exigeaient que l'on ne traduise pas toujours littéralement les titres de recettes, que Lin Hong a souvent voulu mystérieux et poétiques.

C'est pourquoi j'ai parfois omis de traduire certains mots, comme par exemple gong « offrande » (Recettes 31, 49), ou au contraire j'ai précisé d'un mot supplémentaire la traduction, en ajoutant par exemple « pâtes » à botuo (Recette 13). De même, j'ai chaque fois que possible adopté une forme standard quand elle existe en français pour désigner une préparation, par exemple «meurette» pour les cuissons au vin (Recettes 47, 75) ou encore « bourgeons de sapin » (Recette 27), appellation considérée comme impropre par les dictionnaires français, dans la mesure où en France comme en Chine c'est le bourgeon de pin que l'on utilise pour ses propriétés thérapeutiques et non celui du sapin. Il n'en reste pas moins que la terminologie a consacré les « bourgeons de sapin » et non les «bourgeons de pin ». De même, l'habitude nous fait dire « jaune poussin » et non comme en chinois « jaune oie », c'est pourquoi ehuang dousheng est rendu par "pousses de soja jaune poussin », le poussin ayant un duvet aussi jaune que l'oison.

Les trois termes chinois désignant une préparation liquide, aujourd'hui appelée « soupe » en français, ont été traduits comme suit : geng « potage », plat liquide à consistance épaisse ; zhou « porridge », préparation céréalière aqueuse ; tang "bouillon », eau bouillante servant à la cuisson ou au pochage d'ingrédients divers. J'évite volontairement l'emploi du mot « soupe ", aujourd'hui mot générique en français de ces préparations, mais qui ne l'est devenu que tardivement ; « soupe » ayant signifié pendant de longs siècles «tranches de pain ou de biscuit » que l'on faisait tremper dans un liquide, avant de désigner le liquide lui-même par un procédé lexical courant jouant sur la métonymie.

À l'époque de Lin Hong, les pâtes alimentaires et les galettes étaient encore appelées du mot générique bing, tandis que le composé tangbing désignait toute espèce de pâtes en bouillon, farcies ou non farcies. Le choix 
de la traduction dans ce cas dépend du contexte, qui indique s'il s'agit de pâtes alimentaires ou de pâtisseries. On trouve néanmoins deux attestations de l'usage de mian au sens de " pâtes alimentaires » dans des titres (Recettes 17 et 62), usage qui prévaudra par la suite, tandis que bing finira par désigner uniquement les galettes et autres gâteaux à base de farine de blé. Pour revenir à ma remarque sur l'usage du mot «soupe », il est vrai que j'aurais pu traduire zhen tangbing par «Véritables soupes » (Recette 40), puisque le titre de cette recette comporte un jeu de mot sur le terme tangbing, dont le sens dénoté est en principe «pâtes en bouillon », mais que le lecteur doit en fait déchiffrer avec son sens analytique : bouillon-galette, puisque dans l'histoire qui est rapportée par Lin Hong l'invité auquel on promet de «Véritables pâtes en bouillon », intrigué, se demande s'il peut exister de « Fausses pâtes en bouillon ». Ce n'est qu'en voyant le contenu de son bol, une galette à l'huile trempée dans du bouillon, qu'il comprend ce que zhen tangbing signifiait. La terminologie des pâtes chinoises à l'époque est extrêmement riche et complexe. Ainsi, tao " cribler, laver le riz en le criblant » désigne en fait un type de pâtes consommées froides après avoir été rincées dans l'eau après cuisson, d'où leur nom (Recette 12). On notera que le mot fen, qui aujourd'hui peut désigner des vermicelles à base d'amidon ou encore l'amidon lui-même, ne qualifie ici que ce dernier produit. Pour plus d'informations sur la question des pâtes sous les Song, cf. F. SabbanServenti, op. cit.

Par ailleurs, le mot jian, qui a deux acceptions selon la sphère de son emploi, «faire frire » et « faire une décoction », a été traduit, en fonction du contexte, par «beignet » (Recette 33) ou « frit » (Recette 64) et par " confiture sèche » (Recette 63), ce dernier choix s'inspirant de la terminologie ancienne de la confiserie en français.

Enfin, le composé hanju, littéralement « préparation froide », traduit ici par « beignet », apparaît pour la première fois dans le Chuci, et désignera par la suite un gâteau dont l'une des caractéristiques, fréquemment signalée dans les textes, est d'être gras, soit parce qu'il contient de la graisse, soit parce qu'il est frit. Le mode de cuisson de la première recette relevée dans le Qimin yaoshu indique bien une friture. Par ailleurs, cette pâtisserie est souvent associée à la Fête du Manger froid (hanshi), d'où probablement son nom. 


\section{Index des recettes citées dans l'article}

1 qingjing fan, 青 精飯 Riz sombre

2 bi jian geng, 碧澗墨美 Potage de la ravine verte

3 muxu pan, 首蕧盤 Plat de germes de luzerne

5 taishou geng, 太守美 Le potage du préfet

6 binghu zhen, 冰壼珍 Délices d'une jarre glacée

7 lantian $y u$, 藍田王 Jade de Lantian

8 douzhou, 豆弹 Porridge aux haricots

10 hanju, 寒具 Beignets

11 huangjin ji, 黃金雞 Poulet jaune d'or

12 huaiye tao, 槐葉淘 Pâtes vertes

13 dihuang botuo, 地黃䬪慨 Pâtes botuo à la rehmannia

14 meihua tangbing, 梅花湯餅 Raviolis fleurs de prunus

15 chungen huntun, 椿根餛餙 Huntun à la racine de cédrèle

16 yu san geng, 玉㭕美 Potage de jade blanc

17 baihe mian, 百合麵 Pâtes au bulbe de lys

18 gualou fen, 括萋粉 Fécule d'anguine

19 su zheng ya, 素蒸鴨 Canard végétarien

21 panglin xian, 芳林鮮 Fraîcheurs de la lisière des bois

22 diaogu fan, 雕菰飯 Riz sauvage

23 jindai geng 錦帶美 Potage aux feuilles de xanthocère

24 bojin zhuyu, 煿金煮玉 L'or rôti et le jade bouilli

25 tuzhi dan, 土芝丹 Pilules de taro

26 linye jiu, 柳葉非 Ciboule de Chine à feuilles lancéolées

27 songhuang bing, 松黃餅 Galettes aux bourgeons de sapin

28 su qiong ye, 酥堖葉 Feuilles de jade croustillantes

29 Yuanxiu cai, 元修荣 Légume de Yuanxiu

30 ziyingju, 紫英菊 Chrysanthèmes

31 yinsi gong, 銀絲供 Fils d'argent

32 fuci fen, 兒茨粉 Fécule de châtaignes d'eau

33 yanbo jian, 䉸薈煎 Beignets de gardénias

34 haolou cai, 高萋荣 Armoises en légume

35 yuguan fei, 王灌肺 Mou de jade farci

36 jin xian cai, 進賢荣 Légume de promotion des gens capables

37 shanhai dou, 山海兒 Chaussons mer-montagne

38 boxia gong, 撥霞供 L'offrande de nuées empourprées

40 zhen tangbing, 真湯餅 Véritables pâtes en bouillon

41 hangxie jiang, 沉滩墏 Liqueur de rosée

43 xiangyuan bei, 香圓杯 Des cédrats pour coupes à boire

44 xie niang cheng, 蟹釀橙 Crabe en orange farcie

45 lianfang yubao, 蓮房魚包 Papillote de poisson en coupe de lotus

47 jiu zhu cai, 酒者荣 Meurette de légumes

49 chiao gong, 持帮供 Se saisir des pinces de crabe

52 jinfan, 金飯 Riz doré

53 baishi geng, 白石美 Potage de pierres blanches 
54 meizhou, 梅弹 Porridge aux fleurs de prunus

55 shanjia san cui, 山家三脆 Les trois choses croquantes du montagnard

57 Dongting $y i$, 洞庭 饐 Palets de Dongting

59 penggao, 蓬糕 Gâteaux de riz à l'armoise blanche

61 ru jicai, 如薺荣 Comme la bourse à pasteur

62 luofu mian, 蘿菔麵 Pâtes aux petites raves

63 maimendong jian, 麥門冬煎 Confiture sèche d'ophiopogon

64 jia jian rou; 假煎肉 Viande frite factice

65 chengyu sheng, 橙王生 Jade à l'orange

66 yuyan suobing, 王延索餅 Pâtes à la fécule d'ignames

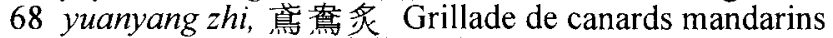

71 ehuang dousheng, 我鳥黃豆生 Pousses de soja jaune poussin

74 man shan xiang, 滿山香 La montagne embaumée

75 jiu zhu yu xun, 酒煮玉䓢 Champignons de jade en meurette

77 shiliu fen, 石榴粉 Perles de grenade

79 heqi zhou, 河祇粥 Porridge au poisson séché

82 Dongpo doufu, 東坡豆腐 Caillé de soja à la $(\mathrm{Su})$ Dongpo

84 yingru $y u$, 賏乳魚 Petits poissons de lait de pavot

85 shengrou jia, 勝肉夾 Raviolis surpassant la viande

89 cui langgan, 脆琅玕 Laitue-asperge croquante

90 zhi zhang, 泉獐 Cerf grillé

92 meihua fu, 梅花脯 Tranches sèches à la fleur de prunus

93 niuweili, 牛尾狸 Chat sauvage

94 jinyu geng, 金王美 Potage d'or et de jade

95 shan zhu yang, 山者羊 Ragoût de mouton à la montagnarde

96 niubang $f u$, 牛膀脯 Bardane séchée

97 mudan shengcai, 牡丹生菜 Les pivoines comme légumes

98 buhan ji, 不寒覒 Choucroute non-froide

99 su xing jiu bing, 素腥酒冰 Gelée de poisson végétarienne pour accompagner le vin 


\section{Caractères chinois}

anxin 安心

Bagualou八卦樓

baihao 白蒿

baipeng白蓬

Bencao 本草

Benxin zhai shushi pu本 心齋蔬食譜

bigu辟穀

bianshi 變食

bu ke he jirou shi zhi, mian fa huang 不可合雞肉食 cai 荣 之面發黃

caise荣色

Cai Zun 蔡遵

chao*炒

chao 焯

chongji 蟲積

Chongxiu Zhenghe jingshi zhenglei beiyong bencao 重修政和經史證類備 用本草

Chuci 楚辭

Dongjing menghua lu 東京 夢華錄

Dongpo rou 東坡肉

Ducheng jisheng 都城紀勝 $\mathrm{Du} \mathrm{Fu}$ 杜甫

fa wu zhi, li xia xue 發五痔

fan 飯

fengchong 風蟲

fu服 (absorber)

fu 賦 (poème)

fugui 賦歸

fu tong da xie 腹痛大瀉

fu yi er wan, ke bu ji 服一 二丸可不饑

geng 慈

gong 供

Gu Ao 顧影

Guiqu lai $x i$ ci 歸去來兮辭 hanju 寒具

hanshi寒食
Hejing weng 和靖翁

Hejing xiansheng 和靖先 生

hongqu 紅麥米

Hu Sihui 忽思慧

huashi 化食

huai junzi 壞君子

Huangdi neijing黄帝内經

Huang Tingjian 黃庭堅

Huo Ru'an 霍如庵

jiang (you) 醬 (油)

Jiangxi lin shanfang shuyuan 江西林山房書院

jin fan 金飯

jin wei chang zhai進䈆長齋

Jinxin 甾心

Jingju淨居

Jingyi 靖逸

jiu 酒

jiu fu, qing shen, chang nian, ling ren bu ji 久服輕身 長年令人不饑

jiu fu, yan nian yi yan 久服 延年盆顏

Jiuhuang bencao 救荒本草 jiu shi, keyi ming mu, yan nian 久食可以明目延 年

junzi 君子

Keshan 可山

kuai 膾

li 痢

Li Bai 李白

Lisao 離騷

liaowu料物

Lin Bu 林逋

Lin Hejing 林和靖

Lin Hong 林洪

Liu Bang 劉邦

LuXun quanji. Qiejieting zawen, yinshi 魯迅全集 且介亭雜文, 隱主

Lu You陸游

Lunyu 論語 
Mengliang lu 夢梁錄

Mengzi孟子

muer木耳

muxu 苜萑

nanzhu mu南燭木

Ning Yuan 凝遠

penghao 蓬蒿

pu ju shan shi 僕居山時

Qimin yaoshu 齊民要術

qing 清

qinggong 清供

qinggonglei 清供類

qing jing mi 青精米

qing shizhi 青石脂

Qingyilu 清異錄

rou sui duo, bu shi sheng shi qi 肉雖多不使勝食氣 ru shan 入山

Rucao jishi 茹草紀事

shan 山

shanfang 山房

shanjia 山家

Shanjia qinggong 山家清供

Shanjia qingshi 山家清事

shanju 山居

shanju xitong 山居系統

Shanju yaoshu山居要術

shanke山客

shanli 山栗

shanlin 山林

shanlin huhai zhi shi

山林湖海之士

shanlin pengyou 山林朋友

shanlin zhi wei 山林之味

shanmin 山民

shanren 山人

shanting山庭

shi 柿 (kaki)

shi 食 (manger)

Shiji 史記

Shidafu shishi wuguan

土大夫食時五觀

shi tun zi jie食豚自戒

shiwu xiangfan 食物相反

$S i k u$ 四庫

su 素
Su (Shi) Dongpo 蘇 (軾) 東 坡

sushi 素食

Su Yijian 蘇易簡

Tang Shenwei 唐慎微

Tang Xiaoxin 湯孝信

Tao Gu陶谷

Tao Yuanming陶淵明

tong xinqi 通心氣

Wang Min 王旻

wenbu溫補

Wu Cunkai 吳存楷

wu fan 烏飯

Wulin jiushi 武林舊事

Wuxing 吳興

Xihu laoren fansheng lu 西 湖老人繁勝錄

xianfang 仙方

Xiangdang 郷黨

xiangliao香料

xiaoren 小人

Xie Yizhai 謝縊齋

Xinfeng jiufa 新豐酒法

(Xinke) Wenfang tuzan 新刻

文房圖贊

xintong 心痛

Xue Lingzhi 薛令之

yanzhi 胭脂

Yang Wanli 楊萬里

yaotong 腰痛

Ye Shi 葉適

Yimen guangdu. Shipin 夷

門廣牘。食品

Yinshan zhengyao 飲膳正 要

Yuan Mei 袁枚

Zhang Liang 張 良

Zhang Yizhai 張一齋

Zhang Yuezhai 張約齊

Zhenglei bencao 證類本草

Zheng Weibin鄭渭濱

zhi 痔 (hémorroïdes)

zhi雉 (faisan)

zhi lin bi治淋閉

zhi tan 止痰

Zhong mei yang he tuji 種梅

養鶴圖記 
La diète parfaite d'un lettré retiré sous les Song du sud

Zhou Zizhi 周紫芝

Zhupo shihua 竹坡詩話

Zhu Xi 朱喜
Zhu Xiao 朱潇

Zifang子房

ziyi 滋益 


\title{
Françoise Sabban
}

\section{Résumé}

Françoise SABBAN : La diète parfaite d'un lettré retiré sous les Song du Sud

Élément indispensable aux côtés des fruits et des légumes d'un régime alimentaire fondé sur les céréales, la viande était en Chine un véritable symbole de gourmandise depuis l'Antiquité. Ceux qui avaient les moyens de manger autre chose que des bouillies de céréales ou des potages de légumes et qui pouvaient se permettre des plats riches et carnés manifestaient ainsi leur opulence et leur appartenance à la classe aisée. S'il leur arrivait de s'abstenir de chair, leur abstinence était ritualisée, en particulier dans certaines circonstances, en signe de deuil ou pour marquer une adhésion à une éthique religieuse. À partir des Song, un style de cuisine dit " maigre » fut systématisé et proposé à titre de régime alimentaire pour certains lettrés qui n'avaient aucune fonction officielle et avaient choisi de vivre retirés du monde. Le texte le plus célèbre consacré à cette cuisine, intitulé Shanjia qinggong (Les vivres simples du montagnard) et écrit par Lin Hong (actif 1241-1252), contient cent quatre recettes raffinées à base de " produits de la montagne », essentiellement des plantes sauvages, des légumes, des céréales et quelques gibiers. La plupart des plats proposés étaient préparés pour recevoir des amis. Dans cet article, l'auteur essaie de comprendre comment cette cuisine, à la fois hygiéniste et morale, s'est imposée à partir des Song, comment elle fut interprétée comme le signe extérieur d'une adhésion volontaire à un mode de vie vertueux, sain et simple, en opposition avec les habitudes somptuaires et ostentatoires des lettrés ordinaires assumant des responsabilités officielles.

\begin{abstract}
Françoise SABban: A Perfect Diet for the Chinese Literati under Song Dynasty

Since Antiquity meat was in China both the symbol of greediness and, represented with vegetables and fruits, a necessary part of the diet based on cereals. For those who could afford to eat more than gruels of corse grains or light vegetable broths, the possibility of including various meat and fish products in the ordinary fare was also a way to express wealth and social status. The fasting of meat was ritualized and accepted in certain circumstances, for instance, for funerals or religious purposes. Under the Song Dynasty a kind of "fast cuisine," which nevertheless allowed a small proportion of animal products, was systematized and proposed as
\end{abstract}


a model diet for those literati who were not directly engaged in world affairs and preferred to live retired or even secluded. The most famous recipes book for this cuisine is the Shanjia qinggong by Lin Hong (fl. 1241-1252) which contains 104 refined recipes based essentially on "mountain products" composed mainly of wild plants, vegetables, cereals, and less often of some venison. These were to be prepared when receiving friends. In this article the author tries to understand how this altogether hygienic and moralistic type of cuisine imposed itself from Song Dynasty on as the external sign of a voluntary adhesion to a virtuous, healthy, and plain life, constrasting with the "somptuous" way of life of the ordinary literati engaged in politics. 